\title{
Inertia-Free Spacecraft Attitude Control with Reaction-Wheel Actuation
}

\author{
Avishai Weiss, Xuebo Yang; \\ Ilya Kolmanovsky $\ddagger$ and Dennis S. Bernstein ${ }^{\S}$ \\ Department of Aerospace Engineering, The University of Michigan, Ann Arbor, MI 48109-2140
}

\begin{abstract}
We extend the inertia-free continuous control law for spacecraft attitude tracking derived in prior work to the case of three axially symmetric reaction wheels. These wheels are assumed to be mounted in a known and linearly independent, but not necessarily orthogonal, configuration with an arbitrary and unknown orientation relative to the spacecraft principal axes. We analyze the stability and performance of the closed-loop system under scale-factor sensor and actuator uncertainty. Finally, we present a numerical study of the algorithm's robustness to sensor noise.
\end{abstract}

\section{Introduction}

In spacecraft applications it is often expensive to determine the mass properties with a high degree of accuracy. To alleviate this requirement, the control algorithms given in refs. ${ }^{1,2}$ require no prior modeling of the mass distribution. These algorithms incorporate internal states that can be viewed as estimates of the moments and products of inertia; however, these estimates need not converge to the true values, and in fact do not converge to the true values except in cases of sufficiently persistent motion.

The results of ref. ${ }^{1}$ are based on rotation matrices in place of quaternions as used in refs. ${ }^{2-4}$ Quaternions provide a double cover of the rotation group $\mathrm{SO}(3)$, and thus, when used as the basis for a continuous control algorithm cause unwinding, that is, unnecessary rotation away from and then back to the desired physical attitude. ${ }^{5}$ To avoid unwinding while using quaternions it is thus necessary to resort to discontinuous control algorithms, which introduce the possibility of chatter due to noise as well as mathematical complications. ${ }^{6-8}$ On the other hand, rotation matrices allow for continuous control laws but introduce multiple equilibria. The additional equilibria that do not represent the desired equilibrium are rendered saddle points by the closed-loop system, and thus present no inherent difficulties.

The goal of this paper is to extend the inertia-free control law developed in ref. ${ }^{1}$ to include reactionwheel actuation and additional realistic effects. We derive the equations of motion for such a satellite and demonstrate the performance of the modified algorithm for slew and spin maneuvers. We then analyze system stability due to scale-factor uncertainty in both the sensors and actuators. Finally, we include the effects of gyro sensor noise.

\section{Spacecraft Dynamics with Reaction Wheels}

In this section we derive the dynamic equations for a spacecraft with reaction wheels, while carefully treating the underlying assumptions on wheel geometry, inertia, and attachment to the spacecraft bus.

We assume that three rotating wheels are attached to a rigid bus as shown in Figure 1. Each wheel is mounted so that it is free to rotate about one of its principal axes passing through its center of mass. For initial generality, however, we do not assume that the mass distribution of each wheel is symmetric about its axis of rotation. For example, the wheels may be elliptical. Let the spacecraft, that is, the bus and wheels,

\footnotetext{
* Graduate student.

${ }^{\dagger}$ Graduate student, Space Control and Inertial Technology Research Center, Harbin Institute of Technology, Harbin, 150001, China. Visiting student at The University of Michigan.

$\ddagger$ Professor, AIAA Member.

$\S$ Professor, AIAA Member.
} 


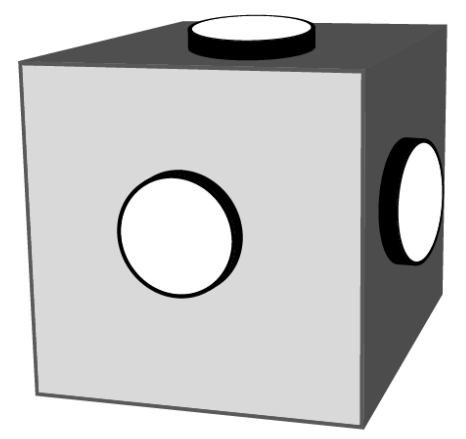

Figure 1: Spacecraft with three reaction wheels.

be denoted by sc, and let c denote its center of mass. Although the spacecraft is not a rigid body, $\mathrm{c}$ is a fixed point, that is, $\mathrm{c}$ does not move relative to the bus. We assume a bus-fixed frame $\mathrm{F}_{\mathrm{B}}$, three wheel-fixed frames $\mathrm{F}_{\mathrm{W}_{i}}$ whose $x$-axes are aligned with the rotation axes of their respective wheels, and an Earth-centered inertial frame $\mathrm{F}_{\mathrm{E}}$. The angular momentum of the spacecraft about its center of mass relative to the inertial frame is given by

$$
\begin{aligned}
\vec{H}_{\mathrm{sc} / \mathrm{c} / \mathrm{E}} & =\vec{H}_{\mathrm{b} / \mathrm{c} / \mathrm{E}}+\sum_{i=1}^{3} \vec{H}_{\mathrm{w}_{i} / \mathrm{c} / \mathrm{E}} \\
& =\vec{I}_{\mathrm{b} / \mathrm{c}} \vec{\omega}_{\mathrm{B} / \mathrm{E}}+\sum_{i=1}^{3} \overrightarrow{\vec{I}}_{\mathrm{w}_{i} / \mathrm{c}} \vec{\omega}_{\mathrm{W}_{i} / \mathrm{E}},
\end{aligned}
$$

where $\overrightarrow{\vec{I}}_{\mathrm{b} / \mathrm{c}}$ is the constant, positive-definite inertia matrix of the bus, that is, the inertia dyadic of the bus relative to the center of mass of the spacecraft, $\vec{I}_{\mathrm{w}_{i} / \mathrm{c}}$ is the constant, positive-definite inertia matrix of wheel $i$ relative to the spacecraft's center of mass, $\vec{\omega}_{\mathrm{B} / \mathrm{E}}$ is the angular velocity of $\mathrm{F}_{\mathrm{B}}$ with respect to $\mathrm{F}_{\mathrm{E}}$, and $\vec{\omega}_{\mathrm{W}_{i} / \mathrm{E}}$ is the angular velocity of wheel $i$ with respect to $\mathrm{F}_{\mathrm{E}}$, which can be decomposed as

$$
\vec{\omega}_{\mathrm{W}_{i} / \mathrm{E}}=\vec{\omega}_{\mathrm{W}_{i} / \mathrm{B}}+\vec{\omega}_{\mathrm{B} / \mathrm{E}}
$$

where $\vec{\omega}_{\mathrm{W}_{i} / \mathrm{B}}$ is the angular velocity of wheel $i$ relative to the bus. Note that $\vec{\omega}_{\mathrm{W}_{i} / \mathrm{B}}$ has only one degree of freedom relative to the bus, which can be seen when resolving it in $\mathrm{F}_{\mathrm{W}_{i}}$, that is,

$$
\left.\vec{\omega}_{\mathrm{W}_{i} / \mathrm{B}}\right|_{\mathrm{W}_{i}}=\left[\begin{array}{c}
q_{i} \\
0 \\
0
\end{array}\right] .
$$

Since $\mathrm{F}_{\mathrm{W}_{i}}$ is aligned with the $i$ th wheel's principal axes, we have

$$
\left.\overrightarrow{\vec{I}}_{\mathrm{w}_{i} / \mathrm{c}}\right|_{\mathrm{W}_{i}}=\left[\begin{array}{ccc}
\alpha_{i} & 0 & 0 \\
0 & \beta_{i} & 0 \\
0 & 0 & \gamma_{i}
\end{array}\right] .
$$

Note that $\vec{\omega}_{\mathrm{W}_{i} / \mathrm{B}}$ is an eigenvector of $\overrightarrow{\vec{I}}_{\mathrm{w}_{i} / \mathrm{c}}$ with eigenvalue $\alpha_{i}$. 


\section{II.A. Equations of Motion}

Before proceeding with control design and simulations, we first derive the equations of motion for a spacecraft actuated with reaction wheels as described above. We begin with Newton's second law for rotation, which states that the derivative of the angular momentum of a body relative to its center of mass in an inertial frame is equal to the sum of the moments applied to that body about its center of mass. We thus have

$$
\begin{aligned}
& \vec{M}_{\mathrm{sc} / \mathrm{c}}=\stackrel{\mathrm{E}}{\vec{H}}_{\mathrm{sc} / \mathrm{c} / \mathrm{E}} \\
& =\overbrace{\overrightarrow{\vec{I}}_{\mathrm{b} / \mathrm{c}} \vec{\omega}_{\mathrm{B} / \mathrm{E}}}^{\mathrm{E} \bullet}+\overbrace{\sum_{i=1}^{3} \overrightarrow{\vec{I}}_{\mathrm{w}_{i} / \mathrm{c}} \vec{\omega}_{\mathrm{W}_{i} / \mathrm{E}}}^{\mathrm{E} \bullet} \\
& =\overbrace{\vec{I}_{\mathrm{b} / \mathrm{c}} \vec{\omega}_{\mathrm{B} / \mathrm{E}}}^{\mathrm{B}}+\vec{\omega}_{\mathrm{B} / \mathrm{E}} \times \overrightarrow{\vec{I}}_{\mathrm{b} / \mathrm{c}} \vec{\omega}_{\mathrm{B} / \mathrm{E}}+\overbrace{\sum_{i=1}^{3} \overrightarrow{\vec{I}}_{\mathrm{w}_{i} / \mathrm{c}} \vec{\omega}_{\mathrm{W}_{i} / \mathrm{E}}}^{\mathrm{B} \bullet}+\vec{\omega}_{\mathrm{B} / \mathrm{E}} \times \sum_{i=1}^{3} \overrightarrow{\vec{I}}_{\mathrm{w}_{i} / \mathrm{c}} \vec{\omega}_{\mathrm{W}_{i} / \mathrm{E}} \\
& =\overrightarrow{\vec{I}}_{\mathrm{b} / \mathrm{c}} \stackrel{\vec{\omega}}{\mathrm{B} / \mathrm{E}}_{\sum_{i=1}^{3} \overrightarrow{\vec{I}}_{\mathrm{w}_{i} / \mathrm{c}} \vec{\omega}_{\mathrm{W}_{i} / \mathrm{E}}}^{\mathrm{B} \bullet}+\vec{\omega}_{\mathrm{B} / \mathrm{E}} \times\left(\overrightarrow{\vec{I}}_{\mathrm{b} / \mathrm{c}} \vec{\omega}_{\mathrm{B} / \mathrm{E}}+\sum_{i=1}^{3} \overrightarrow{\vec{I}}_{\mathrm{w}_{i} / \mathrm{c}} \vec{\omega}_{\mathrm{W}_{i} / \mathrm{E}}\right) .
\end{aligned}
$$

Furthermore,

$$
\begin{aligned}
& \overbrace{\overrightarrow{\vec{I}}_{\mathrm{w}_{i} / \mathrm{c}} \vec{\omega}_{\mathrm{W}_{i} / \mathrm{E}}}^{\mathrm{B} \boldsymbol{}}=\overbrace{\overrightarrow{\vec{I}}_{\mathrm{w}_{i} / \mathrm{c}} \vec{\omega}_{\mathrm{W}_{i} / \mathrm{E}}}^{\mathrm{W}_{i} \bullet}+\vec{\omega}_{\mathrm{W}_{i} / \mathrm{B}} \times \overrightarrow{\vec{I}}_{\mathrm{w}_{i} / \mathrm{c}} \vec{\omega}_{\mathrm{W}_{i} / \mathrm{E}} \\
& =\overrightarrow{\vec{I}}_{\mathrm{w}_{i} / \mathrm{c}} \stackrel{\mathrm{W}_{i} \bullet}{\vec{\omega}} \mathrm{W}_{i / \mathrm{E}}+\vec{\omega}_{\mathrm{W}_{i} / \mathrm{B}} \times \overrightarrow{\vec{I}}_{\mathrm{w}_{i} / \mathrm{c}} \vec{\omega}_{\mathrm{W}_{i} / \mathrm{E}}
\end{aligned}
$$

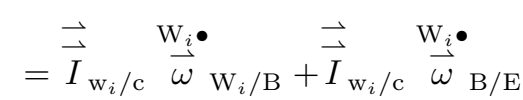

$$
\begin{aligned}
& +\vec{\omega}_{\mathrm{W}_{i} / \mathrm{B}} \times\left(\overrightarrow{\vec{I}}_{\mathrm{w}_{i} / \mathrm{c}} \vec{\omega}_{\mathrm{W}_{i} / \mathrm{B}}+\overrightarrow{\vec{I}}_{\mathrm{w}_{i} / \mathrm{c}} \vec{\omega}_{\mathrm{B} / \mathrm{E}}\right) \\
& =\alpha_{i} \stackrel{\mathrm{B} \bullet}{\vec{\omega}}_{\mathrm{W}_{i} / \mathrm{B}}+\overrightarrow{\vec{I}}_{\mathrm{w}_{i} / \mathrm{c}}\left({\stackrel{\mathrm{B} \bullet}{\vec{\omega}_{\mathrm{B} / \mathrm{E}}}}+\vec{\omega}_{\mathrm{B} / \mathrm{W}_{i}} \times \vec{\omega}_{\mathrm{B} / \mathrm{E}}\right) \\
& +\vec{\omega}_{\mathrm{W}_{i} / \mathrm{B}} \times\left(\alpha_{i} \vec{\omega}_{\mathrm{W}_{i} / \mathrm{B}}+\overrightarrow{\vec{I}}_{\mathrm{w}_{i} / \mathrm{c}} \vec{\omega}_{\mathrm{B} / \mathrm{E}}\right) \\
& =\alpha_{i} \stackrel{\mathrm{B}}{\vec{\omega}}_{\mathrm{W}_{i} / \mathrm{B}}+\overrightarrow{\vec{I}}_{\mathrm{w}_{i} / \mathrm{c}} \stackrel{\mathrm{B} \bullet}{\vec{\omega}}_{\mathrm{B} / \mathrm{E}}-\overrightarrow{\vec{I}}_{\mathrm{w}_{i} / \mathrm{c}}\left(\vec{\omega}_{\mathrm{W}_{i} / \mathrm{B}} \times \vec{\omega}_{\mathrm{B} / \mathrm{E}}\right) \\
& +\vec{\omega}_{\mathrm{W}_{i} / \mathrm{B}} \times \overrightarrow{\vec{I}}_{\mathrm{w}_{i} / \mathrm{c}} \vec{\omega}_{\mathrm{B} / \mathrm{E}} .
\end{aligned}
$$


Substituting (6) into (5), yields a coordinate-free equation that describes the dynamics of the spacecraft given by

$$
\begin{aligned}
\vec{M}_{\mathrm{sc} / \mathrm{c}}= & \vec{I}_{\mathrm{b} / \mathrm{c}} \overrightarrow{\vec{\omega}}_{\mathrm{B} / \mathrm{E}}^{\mathrm{B}}+\sum_{i=1}^{3} \overrightarrow{\vec{I}}_{\mathrm{w}_{i} / \mathrm{c}} \stackrel{\vec{\omega}}{\mathrm{B} / \mathrm{E}}^{\mathrm{B}}+\sum_{i=1}^{3} \alpha_{i}{\stackrel{\vec{\omega}}{\mathrm{W}_{i} / \mathrm{B}}}^{\mathrm{\bullet}} \\
& +\vec{\omega}_{\mathrm{B} / \mathrm{E}} \times\left(\overrightarrow{\vec{I}}_{\mathrm{b} / \mathrm{c}} \vec{\omega}_{\mathrm{B} / \mathrm{E}}+\sum_{i=1}^{3} \overrightarrow{\vec{I}}_{\mathrm{w}_{i} / \mathrm{c}} \vec{\omega}_{\mathrm{W}_{i} / \mathrm{E}}\right) \\
& +\sum_{i=1}^{3} \vec{\omega}_{\mathrm{W}_{i} / \mathrm{B}} \times \overrightarrow{\vec{I}}_{\mathrm{w}_{i} / \mathrm{c}} \vec{\omega}_{\mathrm{B} / \mathrm{E}}-\sum_{i=1}^{3} \overrightarrow{\vec{I}}_{\mathrm{w}_{i} / \mathrm{c}}\left(\vec{\omega}_{\mathrm{W}_{i} / \mathrm{B}} \times \vec{\omega}_{\mathrm{B} / \mathrm{E}}\right) \\
= & \left(\overrightarrow{\vec{I}}_{\mathrm{b} / \mathrm{c}}+\sum_{i=1}^{3} \overrightarrow{\vec{I}}_{\mathrm{w}_{i} / \mathrm{c}}\right) \overrightarrow{\vec{\omega}}_{\mathrm{B} / \mathrm{E}}^{\mathrm{B}}+\sum_{i=1}^{3} \alpha_{i}{\stackrel{\vec{\omega}}{\mathrm{W}_{i} / \mathrm{B}}} \\
& +\vec{\omega}_{\mathrm{B} / \mathrm{E}} \times\left(\left(\overrightarrow{\vec{I}}_{\mathrm{b} / \mathrm{c}}+\sum_{i=1}^{3} \overrightarrow{\vec{I}}_{\mathrm{w}_{i} / \mathrm{c}}\right) \vec{\omega}_{\mathrm{B} / \mathrm{E}}+\sum_{i=1}^{3} \alpha_{i} \vec{\omega}_{\mathrm{W}_{i} / \mathrm{B}}\right) \\
& +\sum_{i=1}^{3}\left(\vec{\omega}_{\mathrm{W}_{i} / \mathrm{B}} \times \overrightarrow{\vec{I}}_{\mathrm{w}_{i} / \mathrm{c}} \vec{\omega}_{\mathrm{B} / \mathrm{E}}-\overrightarrow{\vec{I}}_{\mathrm{w}_{i} / \mathrm{c}}\left(\vec{\omega}_{\mathrm{W}_{i} / \mathrm{B}} \times \vec{\omega}_{\mathrm{B} / \mathrm{E}}\right)\right) .
\end{aligned}
$$

We now resolve (7) in $\mathrm{F}_{\mathrm{B}}$ using the notation

$$
\begin{array}{rlr}
\left.J_{\mathrm{b}} \triangleq \overrightarrow{\vec{I}}_{\mathrm{b} / \mathrm{c}}\right|_{\mathrm{B}}, & \left.J_{\mathrm{w}_{i}} \triangleq \overrightarrow{\vec{I}}_{\mathrm{w}_{i} / \mathrm{c}}\right|_{\mathrm{B}}, \\
\left.J_{\mathrm{w}} \triangleq \sum_{i=1}^{3} \overrightarrow{\vec{I}}_{\mathrm{w}_{i} / \mathrm{c}}\right|_{\mathrm{B}}, & J_{\mathrm{sc}} \triangleq J_{\mathrm{b}}+J_{\mathrm{w}}, \\
\left.\omega \triangleq \vec{\omega}_{\mathrm{B} / \mathrm{E}}\right|_{\mathrm{B}}, & \left.\dot{\omega} \triangleq \overrightarrow{\vec{\omega}}_{\mathrm{B} / \mathrm{E}}\right|_{\mathrm{B}}, \\
\left.\nu_{i} \triangleq \vec{\omega}_{\mathrm{W}_{i} / \mathrm{B}}\right|_{\mathrm{B}}, & \left.\dot{\nu}_{i} \triangleq \overrightarrow{\vec{\omega}}_{\mathrm{W}_{i} / \mathrm{B}}\right|_{\mathrm{B}}, \\
\left.\tau_{\mathrm{dist}} \triangleq \vec{M}_{\mathrm{sc} / \mathrm{c}}\right|_{\mathrm{B}} . &
\end{array}
$$

We let the vector $\tau_{\text {dist }}$ represent disturbance torques, that is, all internal and external torques applied to the spacecraft aside from control torques. Disturbance torques may be due to onboard components, gravity gradients, solar pressure, atmospheric drag, or the ambient magnetic field. 
Resolving (7) in $\mathrm{F}_{\mathrm{B}}$ yields

$$
\begin{aligned}
\tau_{\text {dist }}=( & \left.J_{\mathrm{b}}+J_{\mathrm{w}}\right) \dot{\omega}+\sum_{i=1}^{3} \alpha_{i} \dot{\nu}_{i}+\omega \times\left(\left(J_{\mathrm{b}}+J_{\mathrm{w}}\right) \omega+\sum_{i=1}^{3} \alpha_{i} \nu_{i}\right) \\
& +\sum_{i=1}^{3}\left(\nu_{i} \times J_{\mathrm{w}_{i}} \omega-J_{\mathrm{w}_{i}}\left(\nu_{i} \times \omega\right)\right) \\
=J_{\mathrm{sc}} \dot{\omega} & +\sum_{i=1}^{3} \alpha_{i} \dot{\nu}_{i}+\omega \times\left(J_{\mathrm{sc}} \omega+\sum_{i=1}^{3} \alpha_{i} \nu_{i}\right) \\
& +\sum_{i=1}^{3}\left(\nu_{i} \times J_{\mathrm{w}_{i}} \omega-J_{\mathrm{w}_{i}}\left(\nu_{i} \times \omega\right)\right) .
\end{aligned}
$$

Rearranging (8) yields equations of motion for a spacecraft with reaction wheels, which have the form

$$
\begin{aligned}
J_{\mathrm{sc}} \dot{\omega}= & \left(J_{\mathrm{sc}} \omega+\sum_{i=1}^{3} \alpha_{i} \nu_{i}\right) \times \omega-\sum_{i=1}^{3} \alpha_{i} \mathcal{O}_{\mathrm{B} / \mathrm{w}_{i}} e_{1} u_{i}+\tau_{\mathrm{dist}} \\
& +\sum_{i=1}^{3}\left(J_{\mathrm{w}_{i}}\left(\nu_{i} \times \omega\right)-\nu_{i} \times J_{\mathrm{w}_{i}} \omega\right), \\
\dot{\nu}_{i}= & \mathcal{O}_{\mathrm{B} / \mathrm{w}_{i}} e_{1} u_{i},
\end{aligned}
$$

where $e_{1}=\left[\begin{array}{ccc}1 & 0 & 0\end{array}\right]^{\mathrm{T}}, u_{i}$ is a scalar control, and $\mathcal{O}_{\mathrm{B} / \mathrm{W}_{i}} \in \mathbb{R}^{3 \times 3}$ is the proper orthogonal matrix (that is, the orientation matrix) that transforms components of a vector resolved in $\mathrm{F}_{\mathrm{W}_{i}}$ into the components of the same vector resolved in $\mathrm{F}_{\mathrm{B}}$.

Note that $-\sum_{i=1}^{3} \alpha_{i} \mathcal{O}_{\mathrm{B} / \mathrm{W}_{i}} e_{1} u_{i}$ is the torque applied to the spacecraft due to the wheels. To physically realize this torque, electric motors must apply moments to their respective wheels in order for the wheels to apply the desired reaction torque to the bus. These moments are given by

$$
\tau_{\text {motor }, i}=J_{\mathrm{w}_{i}} \dot{\omega}+\alpha_{i} u_{i} .
$$

The control inputs $u_{i}(t)$ are designed based on equations (9) and (10), and, once they are determined, equation (11) is used to calculate the required torque commands to each motor.

\section{II.B. Special case: symmetric top}

The following result specializes the dynamic equations (9)-(10) to the situation where each wheel is a symmetric top, that is, axially symmetric about its axis of rotation.

Proposition 1. Let $\beta_{i}=\gamma_{i}$ so that

$$
\left.\overrightarrow{\vec{I}}_{\mathrm{w}_{i} / \mathrm{c}}\right|_{\mathrm{W}_{i}}=\left[\begin{array}{ccc}
\alpha_{i} & 0 & 0 \\
0 & \beta_{i} & 0 \\
0 & 0 & \beta_{i}
\end{array}\right] .
$$

Then,

$$
\vec{\omega}_{\mathrm{W}_{i} / \mathrm{B}} \times \overrightarrow{\vec{I}}_{\mathrm{w}_{i} / \mathrm{c}} \vec{\omega}_{\mathrm{B} / \mathrm{E}}-\overrightarrow{\vec{I}}_{\mathrm{w}_{i} / \mathrm{c}}\left(\vec{\omega}_{\mathrm{W}_{i} / \mathrm{B}} \times \vec{\omega}_{\mathrm{B} / \mathrm{E}}\right)=0
$$


Proof. Resolving (12) in $\mathrm{F}_{\mathrm{W}_{i}}$ yields

$$
\begin{aligned}
& \left.\left(\vec{\omega}_{\mathrm{W}_{i} / \mathrm{B}} \times \overrightarrow{\vec{I}}_{\mathrm{w}_{i} / \mathrm{c}} \vec{\omega}_{\mathrm{B} / \mathrm{E}}-\overrightarrow{\vec{I}}_{\mathrm{w}_{i} / \mathrm{c}}\left(\vec{\omega}_{\mathrm{W}_{i} / \mathrm{B}} \times \vec{\omega}_{\mathrm{B} / \mathrm{E}}\right)\right)\right|_{\mathrm{W}_{i}} \\
& =\left[\begin{array}{c}
q_{i} \\
0 \\
0
\end{array}\right] \times\left[\begin{array}{ccc}
\alpha_{i} & 0 & 0 \\
0 & \beta_{i} & 0 \\
0 & 0 & \beta_{i}
\end{array}\right]\left[\begin{array}{c}
\omega_{1} \\
\omega_{2} \\
\omega_{3}
\end{array}\right]-\left[\begin{array}{ccc}
\alpha_{i} & 0 & 0 \\
0 & \beta_{i} & 0 \\
0 & 0 & \beta_{i}
\end{array}\right]\left(\left[\begin{array}{c}
q_{i} \\
0 \\
0
\end{array}\right] \times\left[\begin{array}{l}
\omega_{1} \\
\omega_{2} \\
\omega_{3}
\end{array}\right]\right) \\
& =\left[\begin{array}{ccc}
0 & 0 & 0 \\
0 & 0 & -q_{i} \\
0 & q_{i} & 0
\end{array}\right]\left[\begin{array}{c}
\alpha_{i} \omega_{1} \\
\beta_{i} \omega_{2} \\
\beta_{i} \omega_{3}
\end{array}\right]-\left[\begin{array}{ccc}
\alpha_{i} & 0 & 0 \\
0 & \beta_{i} & 0 \\
0 & 0 & \beta_{i}
\end{array}\right]\left[\begin{array}{ccc}
0 & 0 & 0 \\
0 & 0 & -q_{i} \\
0 & q_{i} & 0
\end{array}\right]\left[\begin{array}{l}
\omega_{1} \\
\omega_{2} \\
\omega_{3}
\end{array}\right] \\
& =\left[\begin{array}{c}
0 \\
-q_{i} \beta_{i} \omega_{3} \\
q_{i} \beta_{i} \omega_{2}
\end{array}\right]-\left[\begin{array}{c}
0 \\
-q_{i} \beta_{i} \omega_{3} \\
q_{i} \beta_{i} \omega_{2}
\end{array}\right]=0 .
\end{aligned}
$$

Henceforth we assume that the wheels are symmetric tops, and thus equations (9)-(10) reduce to

$$
\begin{aligned}
J_{\mathrm{sc}} \dot{\omega} & =\left(J_{\mathrm{sc}} \omega+\sum_{i=1}^{3} \alpha_{i} \nu_{i}\right) \times \omega-\sum_{i=1}^{3} \alpha_{i} \mathcal{O}_{\mathrm{B} / \mathrm{W}_{i}} e_{1} u_{i}+\tau_{\mathrm{dist}}, \\
\dot{\nu}_{i} & =\mathcal{O}_{\mathrm{B} / \mathrm{W}_{i}} e_{1} u_{i} .
\end{aligned}
$$

\section{II.C. Special case: orthogonal wheels aligned with $\mathrm{F}_{B}$}

We further specialize the equations of motion by assuming that the wheels are not only symmetric tops but are also mounted orthogonally to each other. For further simplification, we align $\mathrm{F}_{\mathrm{B}}$ with the rotational axes of the three orthogonal wheels. However, we do not assume that $F_{B}$ is aligned with the principal axes of the bus. In this configuration we have

$$
\mathcal{O}_{\mathrm{B} / \mathrm{W}_{1}} e_{1}=\left[\begin{array}{l}
1 \\
0 \\
0
\end{array}\right], \quad \mathcal{O}_{\mathrm{B} / \mathrm{W}_{2}} e_{1}=\left[\begin{array}{l}
0 \\
1 \\
0
\end{array}\right], \quad \mathcal{O}_{\mathrm{B} / \mathrm{W}_{3}} e_{1}=\left[\begin{array}{l}
0 \\
0 \\
1
\end{array}\right] .
$$

By writing

$$
\begin{aligned}
\sum_{i=1}^{3} \alpha_{i} \mathcal{O}_{\mathrm{B} / \mathrm{W}_{i}} e_{1} u_{i} & =\alpha_{1}\left[\begin{array}{c}
u_{1} \\
0 \\
0
\end{array}\right]+\alpha_{2}\left[\begin{array}{c}
0 \\
u_{2} \\
0
\end{array}\right]+\alpha_{3}\left[\begin{array}{c}
0 \\
0 \\
u_{3}
\end{array}\right] \\
& =\left[\begin{array}{ccc}
\alpha_{1} & 0 & 0 \\
0 & \alpha_{2} & 0 \\
0 & 0 & \alpha_{3}
\end{array}\right]\left[\begin{array}{l}
u_{1} \\
u_{2} \\
u_{3}
\end{array}\right]
\end{aligned}
$$


we can define

$$
J_{\alpha} \triangleq\left[\begin{array}{ccc}
\alpha_{1} & 0 & 0 \\
0 & \alpha_{2} & 0 \\
0 & 0 & \alpha_{3}
\end{array}\right], \quad u \triangleq\left[\begin{array}{c}
u_{1} \\
u_{2} \\
u_{3}
\end{array}\right]
$$

and the equations of motion (13) and (14) simplify to

$$
\begin{aligned}
J_{\mathrm{sc}} \dot{\omega} & =\left(J_{\mathrm{sc}} \omega+J_{\alpha} \nu\right) \times \omega-J_{\alpha} u+\tau_{\text {dist }}, \\
\dot{\nu} & =u .
\end{aligned}
$$

\section{II.D. Special case: principal axis alignment}

A further special case is considered in ref. ${ }^{14}$ In this case it is assumed that the principal axes of the bus are aligned with the rotational axes of the wheels, that the wheels are mass-balanced relative to the center of mass of the bus so that the center of mass of the spacecraft coincides with the center of mass of the bus, and, finally, that the moments of inertia $\beta_{i}$ of the wheels are lumped into the bus inertia $J_{\mathrm{b}}$. With these assumptions, equations (17) and (18) reduce to equations (7.59) and (7.60) of ref. ${ }^{14}$

For the control laws (30) and (38) given below, we do not need to make these assumptions. Specifically, we only assume that the wheels are symmetric tops and mounted orthogonally to each other, but are not necessarily aligned with the principal axes of the bus.

\section{Spacecraft Model, Assumptions, and Objectives for Control Design}

The kinematics of the spacecraft model are given by Poisson's equation

$$
\dot{R}=R \omega^{\times},
$$

which complements (17) and (18). In (19), $\omega^{\times}$denotes the skew-symmetric matrix of $\omega$, and $R \in \mathbb{R}^{3 \times 3}$ is the rotation dyadic that transforms $\mathrm{F}_{\mathrm{E}}$ into $\mathrm{F}_{\mathrm{B}}$ resolved in either $\mathrm{F}_{\mathrm{E}}$ or $\mathrm{F}_{\mathrm{B}}$. Therefore, $R$ is the proper orthogonal matrix (that is, the rotation matrix) that transforms the components of a vector resolved in the bus-fixed frame into the components of the same vector resolved in the inertial frame.

Compared to the case of thrusters treated in ref., ${ }^{1}$ the reaction wheel actuation complicates the dynamic equations due to the term $J_{\alpha} \nu$ added to (17), as well as the integrators (18) augmented to the system. The kinematic relation (19) remains unchanged. The torque inputs applied to each reaction wheel are limited due to current limitations on the electric motors and amplifiers as well as angular velocity constraints on the wheels.

Both rate (inertial) and attitude (noninertial) measurements are assumed to be available. Gyro measurements $y_{\text {rate }} \in \mathbb{R}^{3}$ are assumed to provide measurements of the angular velocity resolved in the spacecraft frame, that is,

$$
y_{\text {rate }}=\omega+v_{\text {rate }},
$$

where $v_{\text {rate }} \in \mathbb{R}^{3}$ represents the presence of noise in the gyro measurements. Attitude is measured indirectly using sensors such as magnetometers or star trackers. The attitude is determined to be

$$
y_{\text {attitude }}=R \text {. }
$$

When attitude measurements are given in terms of an alternative attitude representation, such as quaternions, Rodrigues's formula can be used to determine the corresponding rotation matrix. Attitude estimation on $\mathrm{SO}(3)$ is considered in ref. ${ }^{13}$

The objective of the attitude control problem is to determine control inputs such that the spacecraft attitude given by $R$ follows a commanded attitude trajectory given by a possibly time-varying $\mathrm{C}^{1}$ rotation matrix $R_{\mathrm{d}}(t)$. For $t \geq 0, R_{\mathrm{d}}(t)$ is given by

$$
\begin{aligned}
\dot{R}_{\mathrm{d}}(t) & =R_{\mathrm{d}}(t) \omega_{\mathrm{d}}(t)^{\times}, \\
R_{\mathrm{d}}(0) & =R_{\mathrm{d} 0},
\end{aligned}
$$


where $\omega_{\mathrm{d}}$ is the desired, possibly time-varying angular velocity. The error between $R(t)$ and $R_{\mathrm{d}}(t)$ is given in terms of the attitude-error rotation matrix

$$
\tilde{R} \triangleq R_{\mathrm{d}}^{\mathrm{T}} R
$$

which satisfies the differential equation

$$
\dot{\tilde{R}}=\tilde{R} \tilde{\omega}^{\times},
$$

where the angular velocity error $\tilde{\omega}$ is defined by

$$
\tilde{\omega} \triangleq \omega-\tilde{R}^{\mathrm{T}} \omega_{\mathrm{d}}
$$

We rewrite (17) in terms of the angular-velocity error as

$$
J_{\mathrm{sc}} \dot{\tilde{\omega}}=\left[J_{\mathrm{sc}}\left(\tilde{\omega}+\tilde{R}^{\mathrm{T}} \omega_{\mathrm{d}}\right)+J_{\alpha} \nu\right] \times\left(\tilde{\omega}+\tilde{R}^{\mathrm{T}} \omega_{\mathrm{d}}\right)+J_{\mathrm{sc}}\left(\tilde{\omega} \times \tilde{R}^{\mathrm{T}} \omega_{\mathrm{d}}-\tilde{R}^{\mathrm{T}} \dot{\omega}_{\mathrm{d}}\right)-J_{\alpha} u+\tau_{\mathrm{dist}} .
$$

A scalar measure of attitude error is given by the rotation angle $\theta(t)$ about an eigenaxis needed to rotate the spacecraft from its attitude $R(t)$ to the desired attitude $R_{\mathrm{d}}(t)$, which is given by ${ }^{12}$

$$
\theta(t)=\cos ^{-1}\left(\frac{1}{2}[\operatorname{tr} \tilde{R}(t)-1]\right) .
$$

\section{Controller Design}

The following preliminary results are needed. Let $I$ denote the identity matrix, whose dimensions are determined by context, and let $M_{i j}$ denote the $i, j$ entry of the matrix $M$. The following result is given in ref. ${ }^{1}$

Lemma 1. Let $A \in \mathbb{R}^{3 \times 3}$ be a diagonal positive-definite matrix. Then the following statements hold for a proper orthogonal matrix $R$ :

i) For all $i, j=1,2,3, R_{i j} \in[-1,1]$.

ii) $\operatorname{tr}(A-A R) \geq 0$.

iii) $\operatorname{tr}(A-A R)=0$ if and only if $R=I$.

For convenience we note that, if $R$ is a rotation matrix and $x, y \in \mathbb{R}^{3}$, then

$$
(R x)^{\times}=R x^{\times} R^{\mathrm{T}},
$$

and, therefore,

$$
R(x \times y)=(R x) \times R y .
$$

Next we introduce the notation

$$
J_{\mathrm{sc}} \omega=L(\omega) \gamma
$$

where $\gamma \in \mathbb{R}^{6}$ is defined by

$$
\gamma \triangleq\left[\begin{array}{llllll}
J_{11} & J_{22} & J_{33} & J_{23} & J_{13} & J_{12}
\end{array}\right]^{\mathrm{T}}
$$

and

$$
L(\omega) \triangleq\left[\begin{array}{cccccc}
\omega_{1} & 0 & 0 & 0 & \omega_{3} & \omega_{2} \\
0 & \omega_{2} & 0 & \omega_{3} & 0 & \omega_{1} \\
0 & 0 & \omega_{3} & \omega_{2} & \omega_{1} & 0
\end{array}\right]
$$

Next, let $\hat{J}_{\mathrm{sc}} \in \mathbb{R}^{3 \times 3}$ denote an estimate of $J_{\mathrm{sc}}$, and define the inertia-estimation error

$$
\tilde{J}_{\mathrm{sc}} \triangleq J_{\mathrm{sc}}-\hat{J}_{\mathrm{sc}} .
$$

8 of 29 
Letting $\hat{\gamma}, \tilde{\gamma} \in \mathbb{R}^{6}$ represent $\hat{J}_{\mathrm{sc}}, \tilde{J}_{\mathrm{sc}}$, respectively, it follows that

$$
\tilde{\gamma}=\gamma-\hat{\gamma}
$$

Likewise, let $\hat{\tau}_{\text {dist }} \in \mathbb{R}^{3}$ denote an estimate of $\tau_{\text {dist }}$, and define the disturbance-estimation error

$$
\tilde{\tau}_{\text {dist }} \triangleq \tau_{\text {dist }}-\hat{\tau}_{\text {dist }} \text {. }
$$

We now summarize the assumptions upon which the following development is based:

Assumption 1. $J_{\mathrm{sc}}$ is constant but unknown.

Assumption 2. $J_{\alpha}$ is constant, nonsingular, and known. That is, we have three orthogonal, symmetric top wheels, and we know the moments of inertia about their spin axes.

Assumption 3. Each component of $\tau_{\text {dist }}$ is a linear combination of constant and harmonic signals, whose frequencies are known but whose amplitudes and phases are unknown.

Assumption 3 implies that $\tau_{\text {dist }}$ can be modeled as the output of an autonomous system of the form

$$
\begin{aligned}
\dot{d} & =A_{d} d, \\
\tau_{\text {dist }} & =C_{d} d,
\end{aligned}
$$

where $A_{d} \in \mathbb{R}^{n_{d} \times n_{d}}$ and $C_{d} \in \mathbb{R}^{3 \times n_{d}}$ are known matrices and $A_{d}$ is a Lyapunov-stable matrix. In this model, $d(0)$ is unknown, which is equivalent to the assumption that the amplitude and phase of each harmonic component of the disturbance is unknown. The matrix $A_{d}$ is chosen to include eigenvalues of all frequency components that may be present in the disturbance signal, where the zero eigenvalue corresponds to a constant disturbance. In effect, the controller provides infinite gain at the disturbance frequency, which results in asymptotic rejection of harmonic disturbance components. In particular, an integral controller provides infinite gain at DC in order to reject constant disturbances. In the case of orbit-dependent disturbances, the frequencies can be estimated from the orbital parameters. Likewise, in the case of disturbances originating from on-board devices, the spectral content of the disturbances may be known. In other cases, it may be possible to estimate the spectrum of the disturbances through signal processing. Assumption 3 implies that $A_{d}$ can be chosen to be skew symmetric, which we do henceforth. Let $\hat{d} \in \mathbb{R}^{n_{d}}$ denote an estimate of $d$, and define the disturbance-state estimation error

$$
\tilde{d} \triangleq d-\hat{d}
$$

Assumptions 1-3 are mathematical idealizations, which we state explicitly in order to provide a precise foundation for the subsequent results. In practice, however, these assumptions can be viewed as approximations, whose validity can be assessed based on engineering judgment.

\section{IV.A. Control Laws}

Two controllers are presented in ref. ${ }^{1}$ The inertia-free control law given by (38) of ref. ${ }^{1}$ requires no modification for the case of reaction wheel actuation. As in ref., ${ }^{1}$ define the Lyapunov candidate

$$
V(\omega, \tilde{R}) \triangleq \frac{1}{2} \omega^{T} J_{\mathrm{sc}} \omega+K_{\mathrm{p}} \operatorname{tr}(A-A \tilde{R}),
$$

where $K_{\mathrm{p}}$ is a positive number and $A \in \mathbb{R}^{3 \times 3}$ is a diagonal positive-definite matrix given by $A=\operatorname{diag}\left(a_{1}, a_{2}, a_{3}\right)$. Let $u$ be given by (38) of ref., ${ }^{1}$ that is,

$$
u=J_{\alpha}^{-1}\left(K_{\mathrm{p}} S+K_{\mathrm{v}} \omega\right),
$$

where $K_{\mathrm{v}} \in \mathbb{R}^{3 \times 3}$ is positive definite, and $S$ is defined as

$$
S \triangleq \sum_{i=1}^{3} a_{i}\left(\tilde{R}^{\mathrm{T}} e_{i}\right) \times e_{i},
$$


where, for $i=1,2,3, e_{i}$ denotes the $i$ th column of the $3 \times 3$ identity matrix. Taking the derivative of (29) along the trajectories of (17) and (18) yields

$$
\begin{aligned}
\dot{V}(\omega, \tilde{R}) & =\omega^{T} J_{\mathrm{sc}} \dot{\omega}+K_{\mathrm{p}} \omega^{T} S \\
& =\omega^{T}\left[\left(J_{\mathrm{sc}} \omega+J_{\alpha} \nu\right) \times \omega-J_{\alpha} u\right]+K_{\mathrm{p}} \omega^{T} S \\
& =\omega^{T}\left(-K_{\mathrm{p}} S-K_{\mathrm{v}} \omega\right)+K_{\mathrm{p}} \omega^{T} S \\
& =-\omega^{T} K_{\mathrm{v}} \omega .
\end{aligned}
$$

Note that we substitute $-J_{\alpha}$ for the input matrix $B$ used in the inertia-free control law (38) of ref. ${ }^{1}$ When no disturbances are present, this controller achieves almost global stabilization of a constant desired configuration $R_{\mathrm{d}}$, that is, a slew maneuver that brings the spacecraft to rest.

A more general control law that tracks a desired attitude trajectory in the presence of disturbances is given by (21) of ref. ${ }^{1}$ This controller is modified for reaction wheel actuators below.

Theorem 1. Let $K_{\mathrm{p}}$ be a positive number, let $K_{1} \in \mathbb{R}^{3 \times 3}$, let $Q \in \mathbb{R}^{6 \times 6}$ and $D \in \mathbb{R}^{n_{d} \times n_{d}}$ be positive definite matrices, let $A=\operatorname{diag}\left(a_{1}, a_{2}, a_{3}\right)$ be a diagonal positive-definite matrix, and define $S$ as in (31). Then the function

$$
V(\tilde{\omega}, \tilde{R}, \tilde{\gamma}, \tilde{d}) \triangleq \frac{1}{2}\left(\tilde{\omega}+K_{1} S\right)^{\mathrm{T}} J_{\mathrm{sc}}\left(\tilde{\omega}+K_{1} S\right)+K_{\mathrm{p}} \operatorname{tr}(A-A \tilde{R})+\frac{1}{2} \tilde{\gamma}^{\mathrm{T}} Q \tilde{\gamma}+\frac{1}{2} \tilde{d}^{\mathrm{T}} D \tilde{d}
$$

is positive definite, that is, $V$ is nonnegative, and $V=0$ if and only if $\tilde{\omega}=0, \tilde{R}=I, \tilde{\gamma}=0$, and $\tilde{d}=0$.

Proof. It follows from statement 2 of Lemma 1 that $\operatorname{tr}(A-A \tilde{R})$ is nonnegative. Hence $V$ is nonnegative. Now suppose that $V=0$. Then, $\tilde{\omega}+K_{1} S=0, \tilde{\gamma}=0$, and $\tilde{d}=0$, and it follows from statement 3 of Lemma 1 that $\tilde{R}=I$, and thus $S=0$. Therefore, $\tilde{\omega}=0$.

Note that (33), which we intend to use as a Lyapunov function, is not positive in all the states, namely, it makes no use of the relative angular velocity of the wheels since wheel-speed regulation is not a goal of the control objective.

Theorem 2. Let $K_{\mathrm{p}}$ be a positive number, let $K_{\mathrm{v}} \in \mathbb{R}^{3 \times 3}, K_{1} \in \mathbb{R}^{3 \times 3}, Q \in \mathbb{R}^{6 \times 6}$, and $D \in \mathbb{R}^{n_{d} \times n_{d}}$ be positive definite matrices, assume that $A_{d}^{\mathrm{T}} D+D A_{d}$ is negative semidefinite, let $A=\operatorname{diag}\left(a_{1}, a_{2}, a_{3}\right)$ be a diagonal positive-definite matrix, define $S$ and $V$ as in Theorem 1, and let $\hat{\gamma}$ and $\hat{d}$ satisfy

$$
\dot{\hat{\gamma}}=Q^{-1}\left[L^{\mathrm{T}}(\omega) \omega^{\times}+L^{\mathrm{T}}\left(K_{1} \dot{S}+\tilde{\omega} \times \omega-\tilde{R}^{\mathrm{T}} \dot{\omega}_{\mathrm{d}}\right)\right]\left(\tilde{\omega}+K_{1} S\right),
$$

where

$$
\dot{S}=\sum_{i=1}^{3} a_{i}\left[\left(\tilde{R}^{\mathrm{T}} e_{i}\right) \times \tilde{\omega}\right] \times e_{i}
$$

and

$$
\begin{aligned}
\dot{\hat{d}} & =A_{d} \hat{d}+D^{-1} C_{d}^{\mathrm{T}}\left(\tilde{\omega}+K_{1} S\right), \\
\hat{\tau}_{\text {dist }} & =C_{d} \hat{d},
\end{aligned}
$$

so that $\hat{\tau}_{\mathrm{dist}}$ is the disturbance torque estimator. Furthermore, consider the control law

$$
u=-J_{\alpha}^{-1}\left(v_{1}+v_{2}+v_{3}\right),
$$

where

$$
\begin{gathered}
v_{1} \triangleq-\left(\hat{J}_{\mathrm{sc}} \omega+J_{\alpha} \nu\right) \times \omega-\hat{J}_{\mathrm{sc}}\left(K_{1} \dot{S}+\tilde{\omega} \times \omega-\tilde{R}^{\mathrm{T}} \dot{\omega}_{\mathrm{d}}\right), \\
v_{2} \triangleq-\hat{\tau}_{\text {dist }},
\end{gathered}
$$


and

$$
v_{3} \triangleq-K_{\mathrm{v}}\left(\tilde{\omega}+K_{1} S\right)-K_{\mathrm{p}} S
$$

Then,

$$
\dot{V}(\tilde{\omega}, \tilde{R}, \tilde{\gamma}, \tilde{d})=-\left(\tilde{\omega}+K_{1} S\right)^{\mathrm{T}} K_{\mathrm{v}}\left(\tilde{\omega}+K_{1} S\right)-K_{\mathrm{p}} S^{\mathrm{T}} K_{1} S+\frac{1}{2} \tilde{d}^{\mathrm{T}}\left(A_{d}^{\mathrm{T}} D+D A_{d}\right) \tilde{d}
$$

is negative semidefinite.

Proof. Noting that

$$
\begin{aligned}
\frac{\mathrm{d}}{\mathrm{d} t} \operatorname{tr}(A-A \tilde{R}) & =-\operatorname{tr} A \dot{\tilde{R}} \\
& =-\operatorname{tr} A\left(\tilde{R} \omega^{\times}-\omega_{\mathrm{d}}^{\times} \tilde{R}\right) \\
& =-\sum_{i=1}^{3} a_{i} e_{i}^{\mathrm{T}}\left(\tilde{R} \omega^{\times}-\omega_{\mathrm{d}}^{\times} \tilde{R}\right) e_{i} \\
& =-\sum_{i=1}^{3} a_{i} e_{i}^{\mathrm{T}} \tilde{R}\left(\omega^{\times}-\tilde{R}^{\mathrm{T}} \omega_{\mathrm{d}}^{\times} \tilde{R}\right) e_{i} \\
& =-\sum_{i=1}^{3} a_{i} e_{i}^{\mathrm{T}} \tilde{R}\left(\omega-\tilde{R}^{\mathrm{T}} \omega_{\mathrm{d}}\right)^{\times} e_{i} \\
& =\sum_{i=1}^{3} a_{i} e_{i}^{\mathrm{T}} \tilde{R} e_{i}^{\times} \tilde{\omega} \\
& =\left[-\sum_{i=1}^{3} a_{i} e_{i} \times \tilde{R}^{\mathrm{T}} e_{i}\right]^{\mathrm{T}} \tilde{\omega} \\
& =\left[\sum_{i=1}^{3} a_{i}\left(\tilde{R}^{\mathrm{T}} e_{i}\right) \times e_{i}\right]^{\mathrm{T}} \tilde{\omega} \\
& =\tilde{\omega}^{\mathrm{T}} S,
\end{aligned}
$$


we have

$$
\begin{aligned}
& \dot{V}(\tilde{\omega}, \tilde{R}, \tilde{\gamma}, \tilde{d})=\left(\tilde{\omega}+K_{1} S\right)^{\mathrm{T}}\left(J_{\mathrm{sc}} \dot{\tilde{\omega}}+J_{\mathrm{sc}} K_{1} \dot{S}\right)-K_{\mathrm{p}} \operatorname{tr} A \dot{\tilde{R}}-\tilde{\gamma}^{\mathrm{T}} Q \dot{\hat{\gamma}}+\tilde{d}^{\mathrm{T}} D \dot{\tilde{d}} \\
& =\left(\tilde{\omega}+K_{1} S\right)^{\mathrm{T}}\left[\left(J_{\mathrm{sc}} \omega+J_{\alpha} \nu\right) \times \omega+J_{\mathrm{sc}}\left(\tilde{\omega} \times \omega-\tilde{R}^{\mathrm{T}} \dot{\omega}_{\mathrm{d}}\right)-J_{\alpha} u+\tau_{\text {dist }}+J_{\mathrm{sc}} K_{1} \dot{S}\right] \\
& +K_{\mathrm{p}} \tilde{\omega}^{\mathrm{T}} S-\tilde{\gamma}^{\mathrm{T}} Q \dot{\hat{\gamma}}+\tilde{d}^{\mathrm{T}} D \dot{\tilde{d}} \\
& =\left(\tilde{\omega}+K_{1} S\right)^{\mathrm{T}}\left[\left(J_{\mathrm{sc}} \omega+J_{\alpha} \nu\right) \times \omega+J_{\mathrm{sc}}\left(K_{1} \dot{S}+\tilde{\omega} \times \omega-\tilde{R}^{\mathrm{T}} \dot{\omega}_{\mathrm{d}}\right)\right. \\
& \left.+v_{1}+v_{2}+v_{3}+\tau_{\text {dist }}\right]+K_{\mathrm{p}} \tilde{\omega}^{\mathrm{T}} S-\tilde{\gamma}^{\mathrm{T}} Q \dot{\hat{\gamma}}+\tilde{d}^{\mathrm{T}} D \dot{\tilde{d}} \\
& =\left(\tilde{\omega}+K_{1} S\right)^{\mathrm{T}}\left[\left(\tilde{J}_{\mathrm{sc}} \omega\right) \times \omega+\tilde{J}_{\mathrm{sc}}\left(K_{1} \dot{S}+\tilde{\omega} \times \omega-\tilde{R}^{\mathrm{T}} \dot{\omega}_{\mathrm{d}}\right)\right] \\
& +\left(\tilde{\omega}+K_{1} S\right)^{\mathrm{T}} \tilde{\tau}_{\text {dist }}-\left(\tilde{\omega}+K_{1} S\right)^{\mathrm{T}} K_{\mathrm{v}}\left(\tilde{\omega}+K_{1} S\right)-K_{\mathrm{p}}\left(\tilde{\omega}+K_{1} S\right)^{\mathrm{T}} S \\
& +K_{\mathrm{p}} \tilde{\omega}^{\mathrm{T}} S-\tilde{\gamma}^{\mathrm{T}} Q \dot{\hat{\gamma}}+\tilde{d}^{\mathrm{T}} D \dot{\tilde{d}} \\
& =\left(\tilde{\omega}+K_{1} S\right)^{\mathrm{T}}\left[L(\omega) \tilde{\gamma} \times \omega+L\left(K_{1} \dot{S}+\tilde{\omega} \times \omega-\tilde{R}^{\mathrm{T}} \dot{\omega}_{\mathrm{d}}\right) \tilde{\gamma}\right] \\
& -\left(\tilde{\omega}+K_{1} S\right)^{\mathrm{T}} K_{\mathrm{v}}\left(\tilde{\omega}+K_{1} S\right)-K_{\mathrm{p}} S^{\mathrm{T}} K_{1} S-\tilde{\gamma}^{\mathrm{T}} Q \dot{\hat{\gamma}} \\
& +\tilde{d}^{\mathrm{T}} C_{d}^{\mathrm{T}}\left(\tilde{\omega}+K_{1} S\right)+\tilde{d}^{\mathrm{T}} D\left[A_{d} \tilde{d}-D^{-1} C_{d}^{\mathrm{T}}\left(\tilde{\omega}+K_{1} S\right)\right] \\
& =-\left(\tilde{\omega}+K_{1} S\right)^{\mathrm{T}} K_{\mathrm{v}}\left(\tilde{\omega}+K_{1} S\right)-K_{\mathrm{p}} S^{\mathrm{T}} K_{1} S-\tilde{\gamma}^{\mathrm{T}} Q \dot{\hat{\gamma}} \\
& +\left(\tilde{\omega}+K_{1} S\right)^{\mathrm{T}}\left[-\omega^{\times} L(\omega)+L\left(K_{1} \dot{S}+\tilde{\omega} \times \omega-\tilde{R}^{\mathrm{T}} \dot{\omega}_{\mathrm{d}}\right)\right] \tilde{\gamma} \\
& +\frac{1}{2} \tilde{d}^{\mathrm{T}}\left(A_{d}^{\mathrm{T}} D+D A_{d}\right) \tilde{d} \\
& =-\left(\tilde{\omega}+K_{1} S\right)^{\mathrm{T}} K_{\mathrm{v}}\left(\tilde{\omega}+K_{1} S\right)-K_{\mathrm{p}} S^{\mathrm{T}} K_{1} S \\
& +\tilde{\gamma}^{\mathrm{T}}\left[-Q \dot{\hat{\gamma}}+\left(L^{\mathrm{T}}(\omega) \omega^{\times}+L^{\mathrm{T}}\left(K_{1} \dot{S}+\tilde{\omega} \times \omega-\tilde{R}^{\mathrm{T}} \dot{\omega}_{\mathrm{d}}\right)\right)\left(\tilde{\omega}+K_{1} S\right)\right] \\
& +\frac{1}{2} \tilde{d}^{\mathrm{T}}\left(A_{d}^{\mathrm{T}} D+D A_{d}\right) \tilde{d} \\
& =-\left(\tilde{\omega}+K_{1} S\right)^{\mathrm{T}} K_{\mathrm{v}}\left(\tilde{\omega}+K_{1} S\right)-K_{\mathrm{p}} S^{\mathrm{T}} K_{1} S+\frac{1}{2} \tilde{d}^{\mathrm{T}}\left(A_{d}^{\mathrm{T}} D+D A_{d}\right) \tilde{d} .
\end{aligned}
$$

Future work will complete the proof for almost global stabilization (that is, Lyapunov stability with almost global convergence) of spacecraft tracking using reaction wheels. The proof will rely on partial stability theory and invariance theorems.

\section{Simulation}

Simulation results are used to illustrate the effectiveness of controllers (30) and (38) in controlling the spacecraft attitude and angular velocity using reaction-wheel actuators. To simulate the slew and spin maneuvers, the following spacecraft parameters are assumed. The bus inertia matrix $J_{\mathrm{b}}$ is given by

$$
J_{\mathrm{b}}=\left[\begin{array}{ccc}
5 & -0.1 & -0.5 \\
-0.1 & 2 & 1 \\
-0.5 & 1 & 3.5
\end{array}\right] \mathrm{kg}-\mathrm{m}^{2},
$$

with principal moments of inertia 1.4947, 3.7997, and $5.2056 \mathrm{~kg}-\mathrm{m}^{2}$. The axes of rotation of the three reaction wheels are aligned with the spacecraft body-fixed frame unit vectors, and we assume that the wheel inertias are given by $J_{\mathrm{w}_{1}}=\operatorname{diag}(1,0.75,0.75) \mathrm{kg}-\mathrm{m}^{2}, J_{\mathrm{w}_{2}}=\operatorname{diag}(0.75,1,0.75) \mathrm{kg}-\mathrm{m}^{2}$, and $J_{\mathrm{w}_{3}}=\operatorname{diag}(0.75,0.75,1)$ $\mathrm{kg}-\mathrm{m}^{2}$.

Let $K_{\mathrm{p}}$ be given by

$$
K_{\mathrm{p}}=\frac{\alpha}{\operatorname{tr} A},
$$

and as in ref., ${ }^{1}$ we let $K_{\mathrm{v}}=K_{\mathrm{v}}(\omega)$ be given by

$$
K_{\mathrm{v}}=\beta\left[\begin{array}{ccc}
\frac{1}{1+\left|\omega_{1}\right|} & 0 & 0 \\
0 & \frac{1}{1+\left|\omega_{2}\right|} & 0 \\
0 & 0 & \frac{1}{1+\left|\omega_{3}\right|}
\end{array}\right],
$$


in order to better handle torque saturation.

Illustrative slew maneuvers and spin maneuvers are considered below.

\section{V.A. Slew Maneuver Using Control Law (30) With No Disturbance}

We use controller (30) for an aggressive slew maneuver, where the objective is to bring the spacecraft from the initial attitude $R_{0}=I_{3}$ and initial angular velocity

$$
\omega(0)=\left[\begin{array}{lll}
1 & -1 & 0.5
\end{array}\right]^{\mathrm{T}} \mathrm{rad} / \mathrm{sec}
$$

to rest $\left(\omega_{\mathrm{d}}=0\right)$ at the desired final orientation $R_{\mathrm{d}}=\operatorname{diag}(1,-1,-1)$, which represents a rotation of 180 degrees about the $x$-axis. We assume that the reaction wheels are initially not spinning relative to the spacecraft, that is,

$$
\nu(0)=\left[\begin{array}{lll}
0 & 0 & 0
\end{array}\right]^{\mathrm{T}} \mathrm{rad} / \mathrm{sec} .
$$

We assume no disturbance is present and set $\alpha=\beta=45$.

Figures 2-4 show, respectively, the attitude error, angular velocity components, and wheel relative angular-velocity components. The spacecraft attitude and angular velocity components are brought close to the desired values in about $10 \mathrm{sec}$.

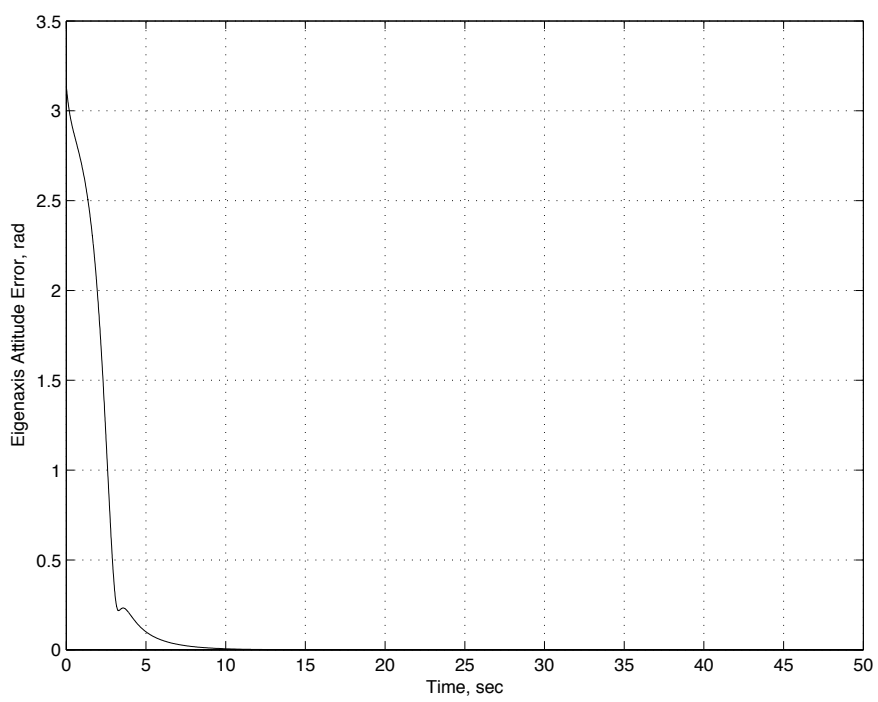

Figure 2: Eigenaxis attitude error for the slew maneuver with momentum wheels. 


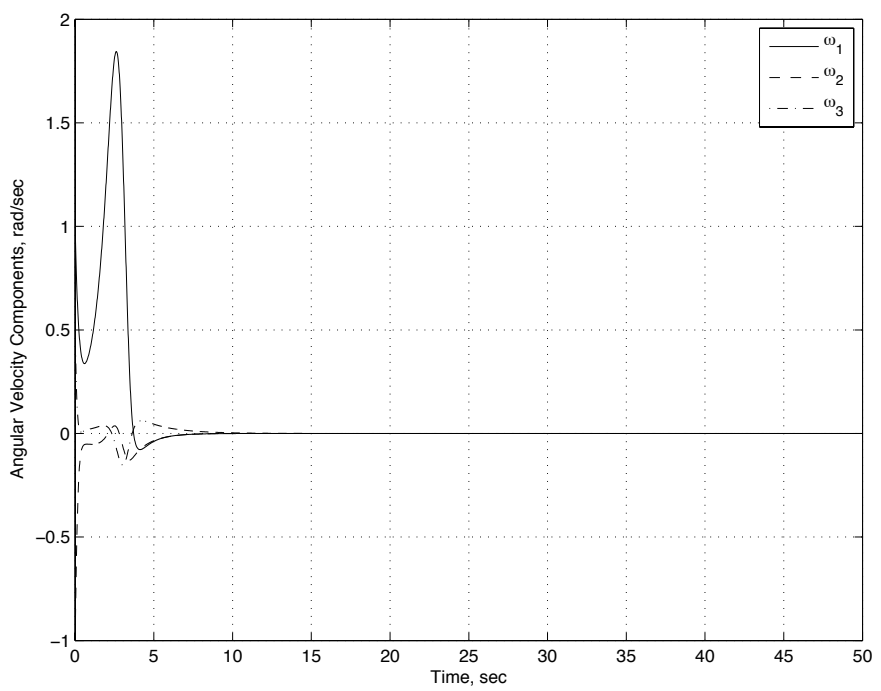

Figure 3: Spacecraft angular-velocity components for the slew maneuver with momentum wheels.

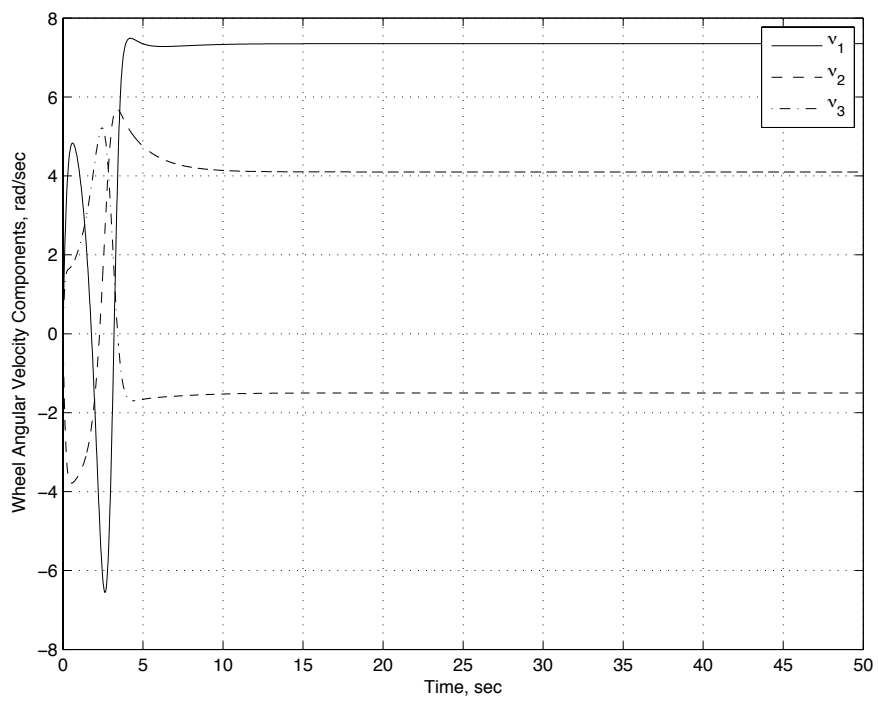

Figure 4: Relative angular-velocity components of the reaction wheels for the slew maneuver. 


\section{V.B. Slew Maneuver Using Control Law (38) Under Constant Disturbance}

We now assume a constant nonzero disturbance torque, $\tau_{\text {dist }}=\left[\begin{array}{lll}0.7 & -0.3 & 0\end{array}\right]^{\mathrm{T}}$. The parameters of controller (38) are chosen to be $K_{1}=I_{3}, A=\operatorname{diag}(1,2,3), \alpha=\beta=1, D=I_{3}$, and $Q=I_{6}$.

Figures 5-10 show, respectively, the attitude error, angular velocity components, wheel relative-angularvelocity components, angular momentum, disturbance-estimate errors, and inertia-estimate errors. The spacecraft attitude and angular velocity components are brought close to the desired values in about 50 sec. Figure 7 indicates that the reaction wheel rotational speed grows unbounded, and thereby increases the total angular momentum of the spacecraft (Figure 8). This behavior is due to the need to compensate for the persistent disturbance torque.

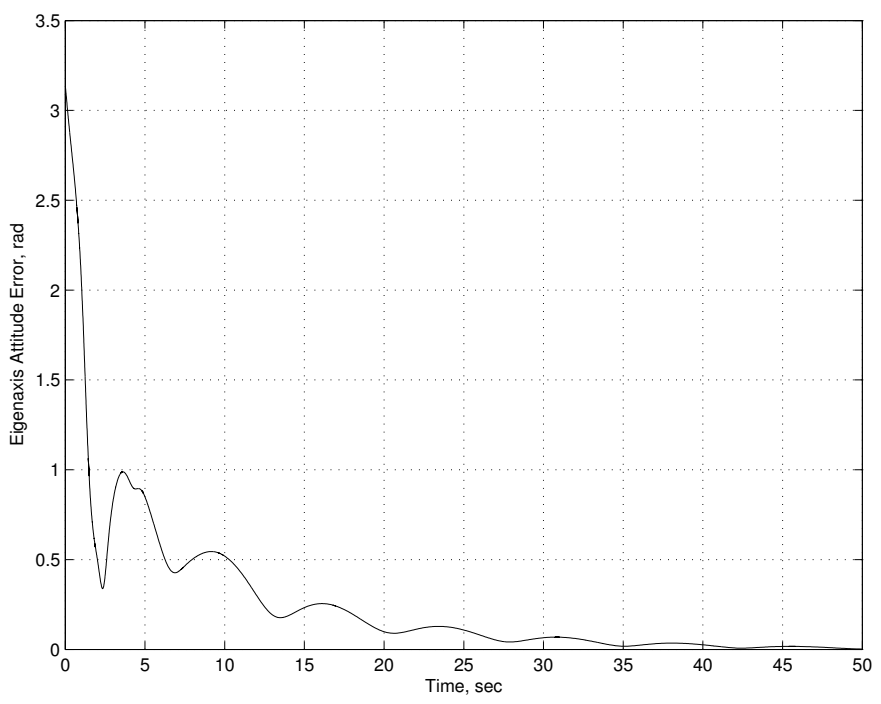

Figure 5: Eigenaxis attitude error for the slew maneuver with momentum wheels.

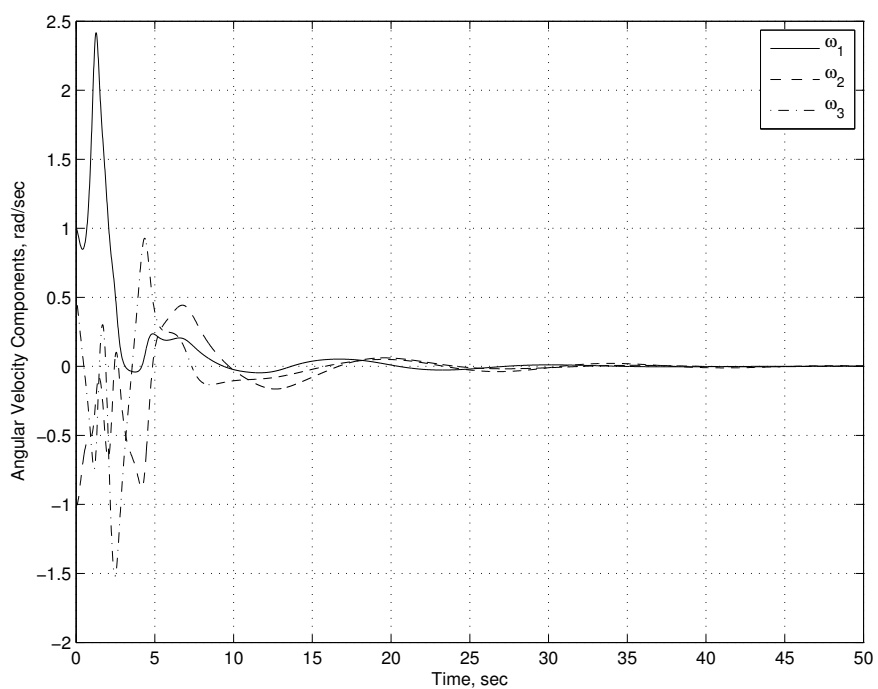

Figure 6: Spacecraft angular-velocity components for the slew maneuver with momentum wheels. 


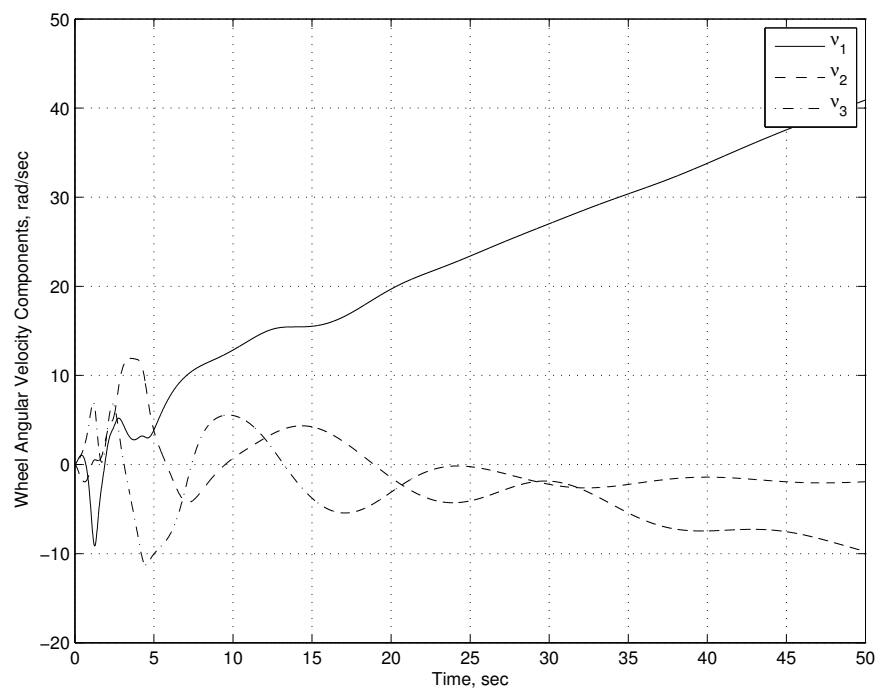

Figure 7: Relative angular-velocity components of the reaction wheels for the slew maneuver. Notice that their spin grows unbounded due to the constant disturbance torque.
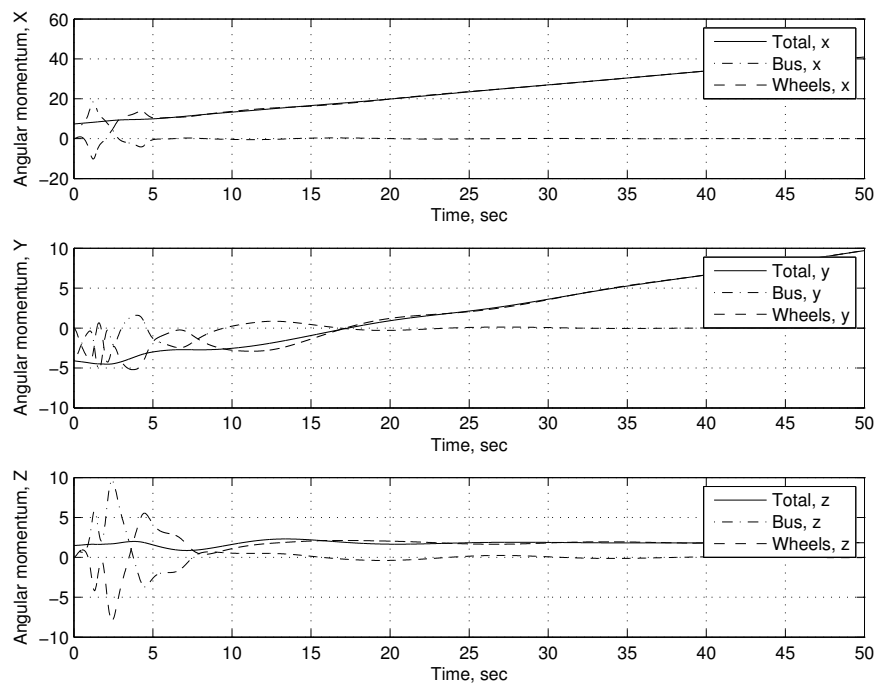

Figure 8: Angular momentum of the spacecraft in the Earth inertial frame for the slew maneuver with momentum wheels. Notice that total angular momentum is not conserved due to the constant disturbance torque. 


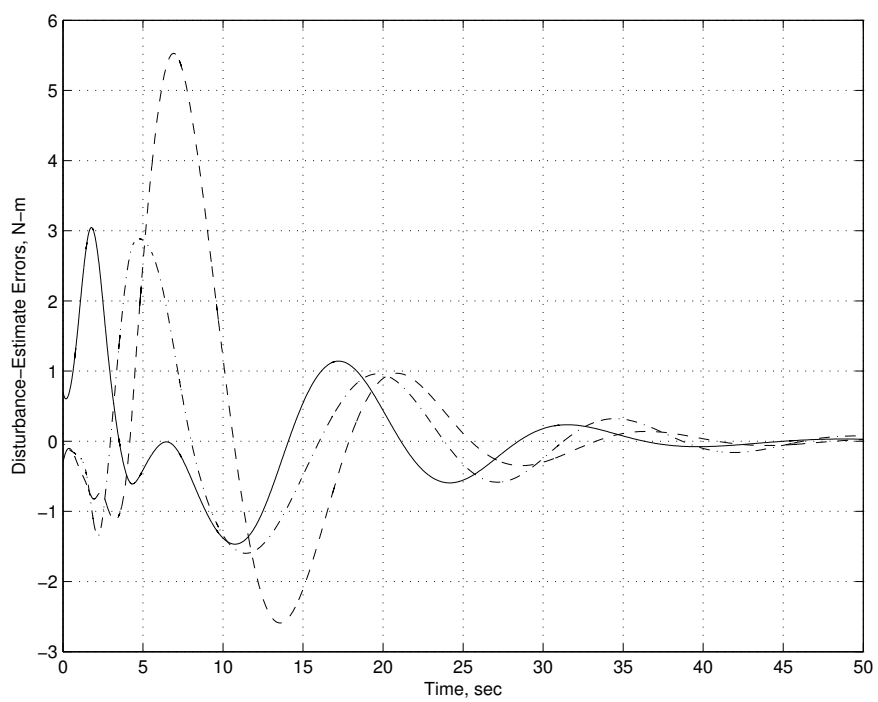

Figure 9: Disturbance-estimate errors for the slew maneuver with momentum wheels.
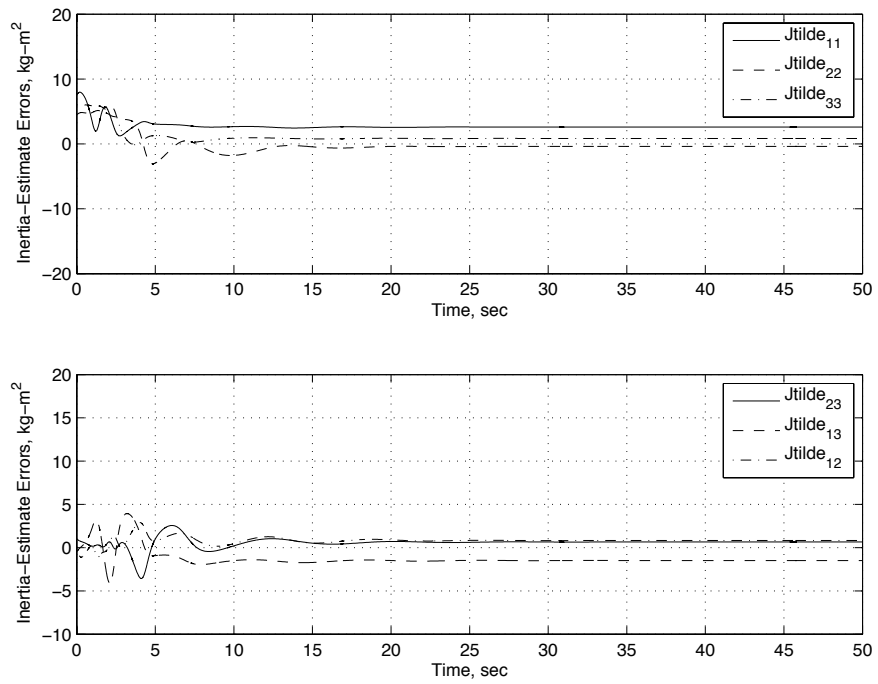

Figure 10: Inertia-estimate errors for the slew maneuver with momentum wheels. 


\section{V.C. Spin Maneuver Using Using Control Law (38)}

We now consider a spin maneuver with the spacecraft initially at rest and $R(0)=I_{3}$. The specified attitude is given by $R_{\mathrm{d}}(0)=I_{3}$ with the desired constant angular velocity

$$
\omega_{\mathrm{d}}=\left[\begin{array}{lll}
0.5 & -0.5 & -0.3
\end{array}\right]^{\mathrm{T}} \mathrm{rad} / \mathrm{sec},
$$

and we assume zero torque disturbance. Figures 11-15 show, respectively, the attitude errors, angular velocity components, wheel relative angular-velocity components, angular momentum, and inertia-estimate errors. For this maneuver, the spin command consists of a specified time history of rotation about a specified body axis aligned in a specified inertial direction. The controller achieves the specified tracking objective within $50 \mathrm{sec}$

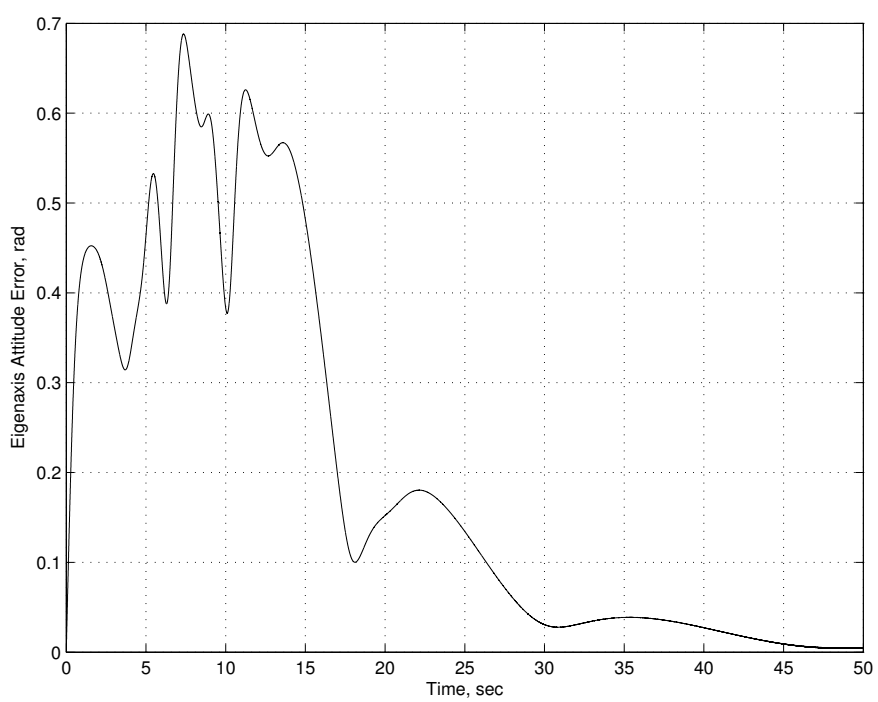

Figure 11: Eigenaxis attitude error for the slew maneuver with momentum wheels.

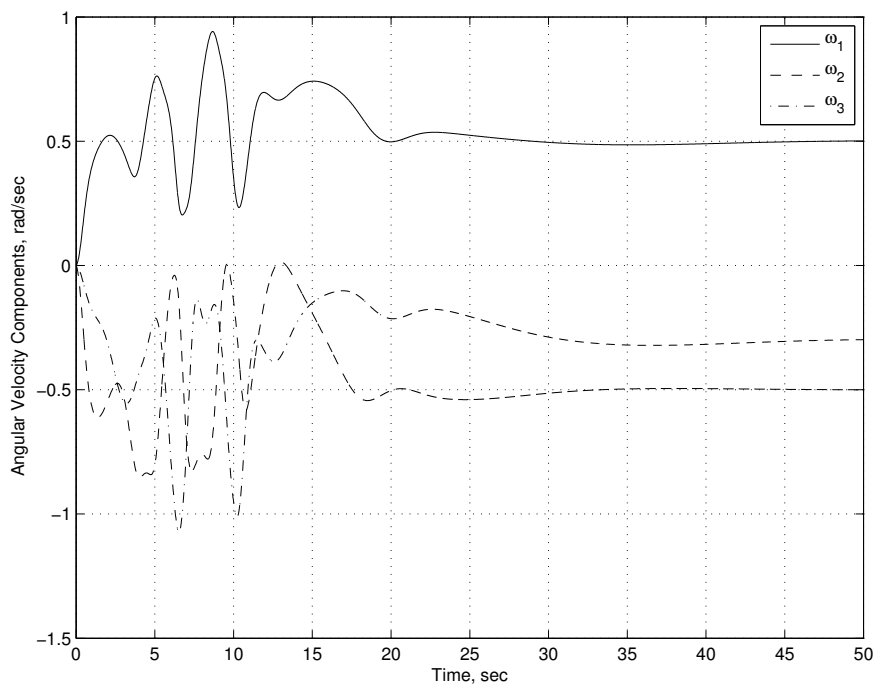

Figure 12: Spacecraft angular-velocity components for the slew maneuver with momentum wheels. 


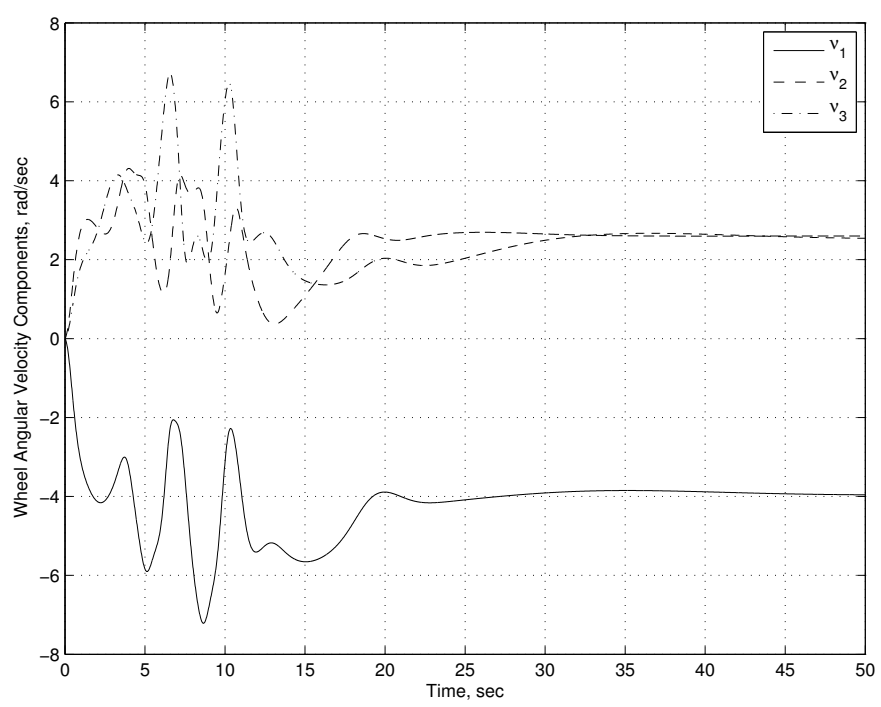

Figure 13: Relative angular-velocity components of the reaction wheels for the slew maneuver.
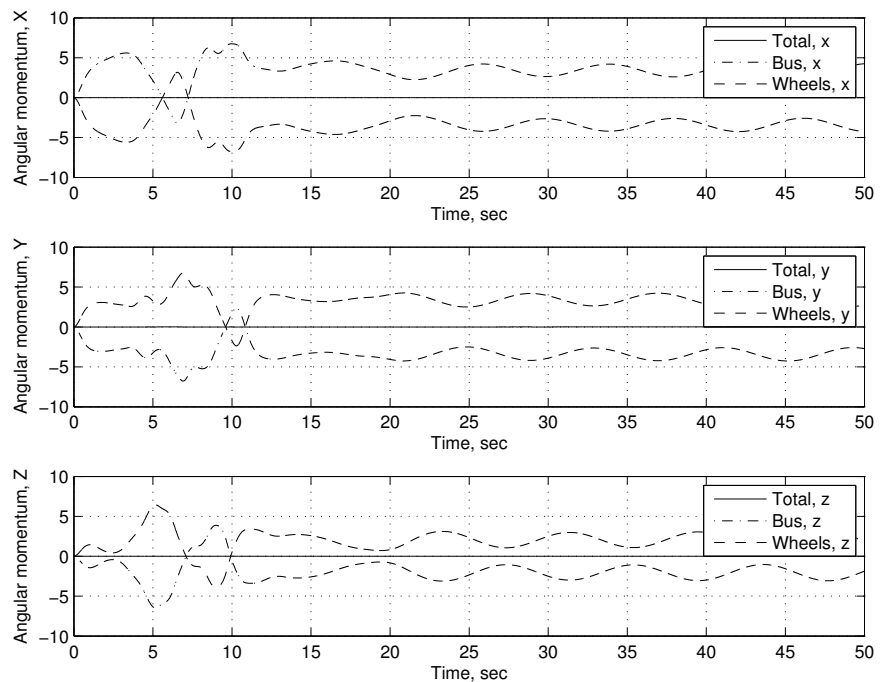

Figure 14: Angular momentum of the spacecraft in the Earth inertial frame for the slew maneuver with momentum wheels. 

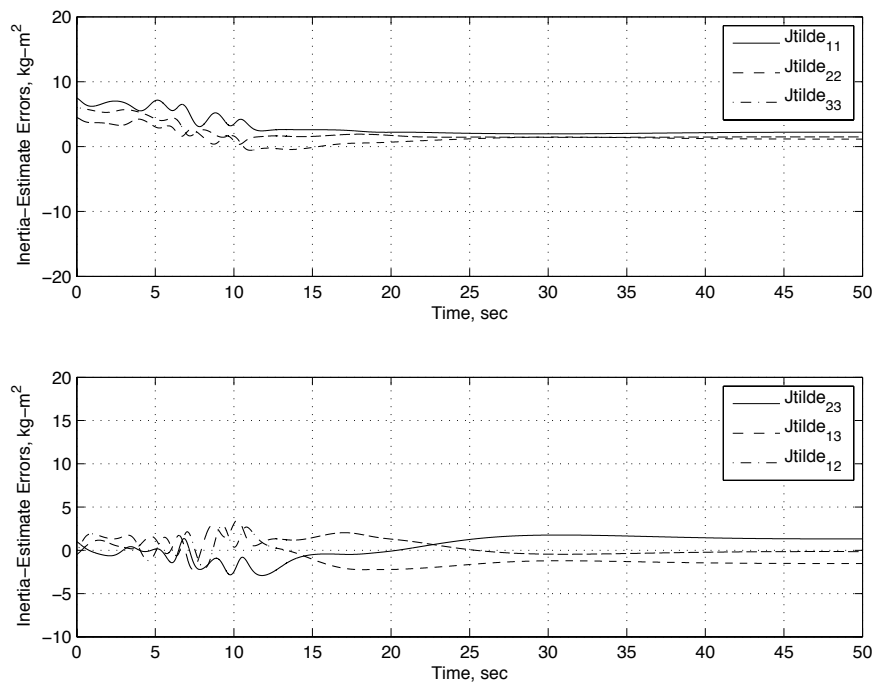

Figure 15: Inertia-estimate errors for the slew maneuver with momentum wheels. 


\section{Scale-Factor Uncertainty in Sensors and Actuators}

We now analyze scale-factor uncertainty in both the sensors and actuators. These uncertainties may arise due to faulty calibration or mechanical failure. We consider the configuration used in Section V.A.

\section{VI.A. Sensor Scale-Factor Uncertainty}

To account for a scale-factor uncertainty in the gyro measurements, we write the control law (30) as

$$
u=-J_{\alpha}^{-1}\left(K_{\mathrm{p}} S+K_{\mathrm{v}} \mu \omega\right),
$$

where $\mu$ is an unknown sensor scale-factor. Using (29) as the Lyapunov candidate, we obtain

$$
\begin{aligned}
\dot{V}(\omega, \tilde{R}) & =\omega^{\mathrm{T}} J_{\mathrm{sc}} \dot{\omega}+K_{\mathrm{p}} \omega^{\mathrm{T}} S \\
& =\omega^{\mathrm{T}}\left[\left(J_{\mathrm{sc}} \omega+J_{\alpha} \nu\right) \times \omega+J_{\alpha} u\right]+K_{\mathrm{p}} \omega^{\mathrm{T}} S \\
& =\omega^{\mathrm{T}}\left(-K_{\mathrm{p}} S-K_{\mathrm{v}} \mu \omega\right)+K_{\mathrm{p}} \omega^{\mathrm{T}} S \\
& =-\omega^{\mathrm{T}} K_{\mathrm{v}} \mu \omega .
\end{aligned}
$$

Note that $\dot{V}$ takes the same form as (32) with an additional scale-factor $\mu$. We can group $\mu$ with the control gain $K_{\mathrm{v}}$, so that for positive values, we reach the same result as before. That is, when no disturbances are present, this controller achieves almost global stabilization of that constant desired configuration $R_{\mathrm{d}}$, which is a slew maneuver that brings the spacecraft to rest.

Figure 16 shows the eigenaxis attitude error and angular-velocity components for representative gyro scale-factors.

\section{VI.B. Actuator Scale-Factor Uncertainty}

For a scale-factor uncertainty in the actuator, we rewrite (17) as

$$
J_{\mathrm{sc}} \dot{\omega}=\left(J_{\mathrm{sc}} \omega+J_{\alpha} \nu\right) \times \omega+J_{\alpha} \mu u,
$$

where $\mu$ is an unknown actuator scale-factor. We modify (29) to include the unknown factor,

$$
V(\omega, \tilde{R}) \triangleq \frac{1}{2} \omega^{\mathrm{T}} J_{\mathrm{sc}} \omega+\mu K_{\mathrm{p}} \operatorname{tr}(A-A \tilde{R}) .
$$

Then, using (30) as the control we obtain

$$
\begin{aligned}
\dot{V}(\omega, \tilde{R}) & =\omega^{\mathrm{T}} J_{\mathrm{sc}} \dot{\omega}+\mu K_{\mathrm{p}} \omega^{\mathrm{T}} S \\
& =\omega^{\mathrm{T}}\left[\left(J_{\mathrm{sc}} \omega+J_{\alpha} \nu\right) \times \omega+J_{\alpha} \mu u\right]+\mu K_{\mathrm{p}} \omega^{\mathrm{T}} S \\
& =\omega^{\mathrm{T}}\left(-\mu K_{\mathrm{p}} S-\mu K_{\mathrm{v}} \omega\right)+\mu K_{\mathrm{p}} \omega^{\mathrm{T}} S \\
& =-\mu \omega^{\mathrm{T}} K_{\mathrm{v}} \omega .
\end{aligned}
$$

As with the case of a gyro scale-factor error, $\dot{V}$ takes the same form as (32). Here $\mu$ modifies both control gains $K_{\mathrm{v}}$ and $K_{\mathrm{p}}$. For positive values of $\mu,(49)$ is positive and the control gains remain valid selections.

Figure 17 shows the eigenaxis attitude error and angular-velocity components for representative actuator scale-factors.

\section{VI.C. Performance}

We consider the performance metric

$$
t_{\mathrm{m}} \triangleq \min \{k>50: \max [\theta(k-i)]<0.1, i=1,2, \ldots, 50 .\},
$$

where $\theta$ is given by (26). Thus, $t_{\mathrm{m}}$ defines the minimum time such that the previous 50 simulation steps all have an eigenaxis attitude error of less than $0.1 \mathrm{rad}$. Figures 18 and 19 show the performance metric versus sensor and actuator scale-factor uncertainty. 


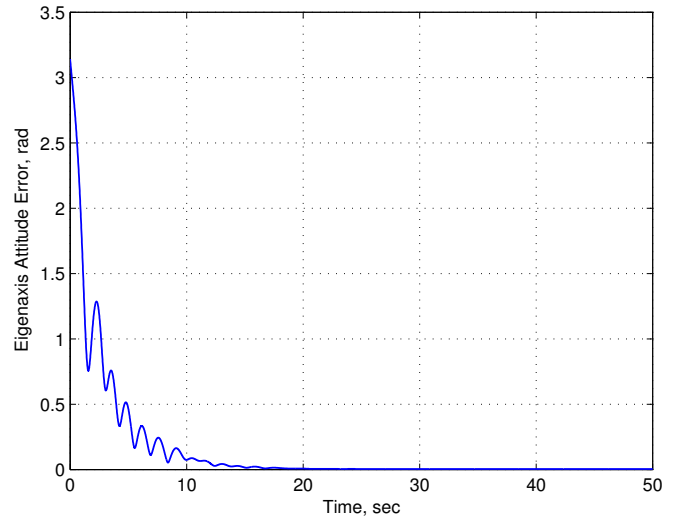

(a) Eigenaxis attitude error with $\mu=0.1$

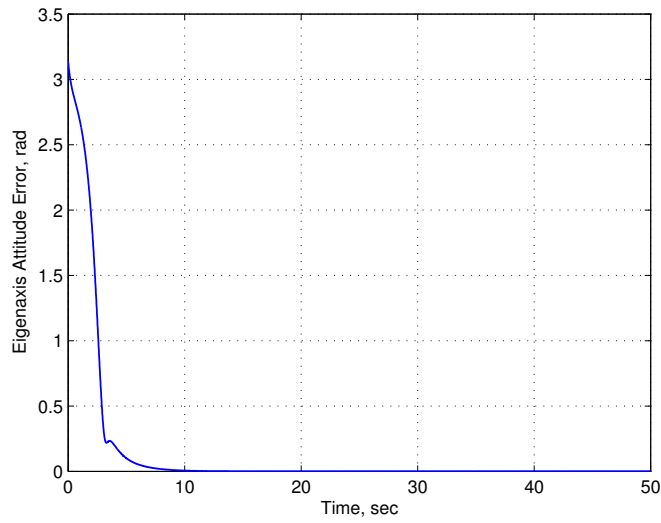

(c) Eigenaxis attitude error with $\mu=1$

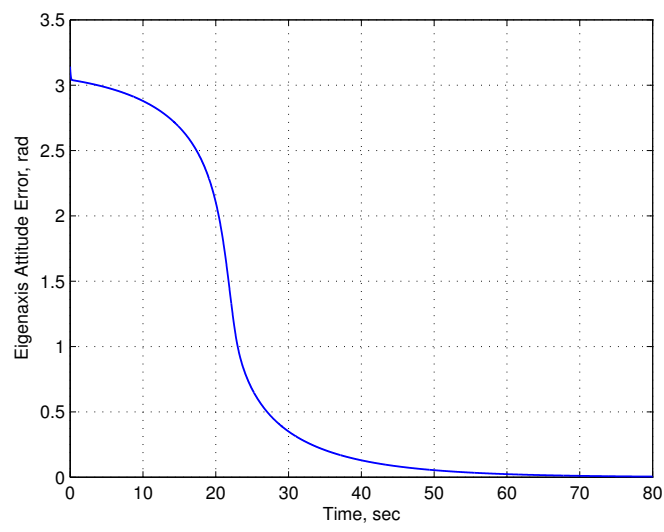

(e) Eigenaxis attitude error with $\mu=10$

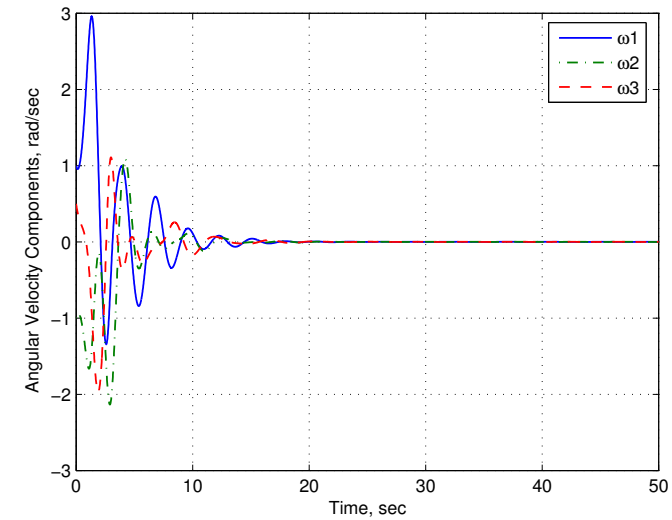

(b) Angular velocity with $\mu=0.1$

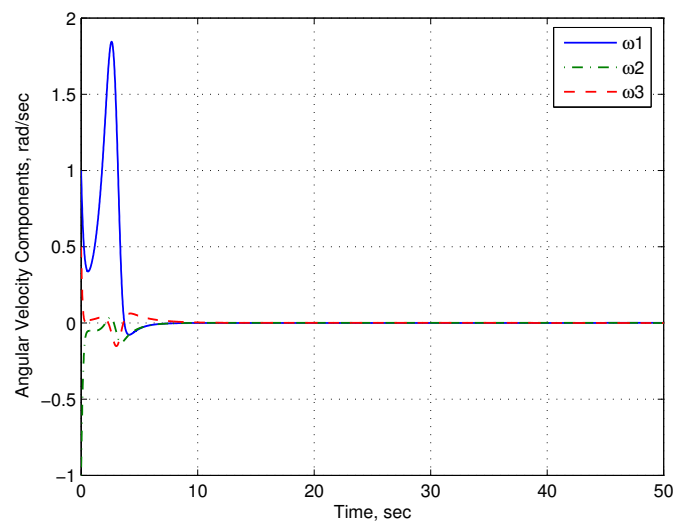

(d) Angular velocity with $\mu=1$

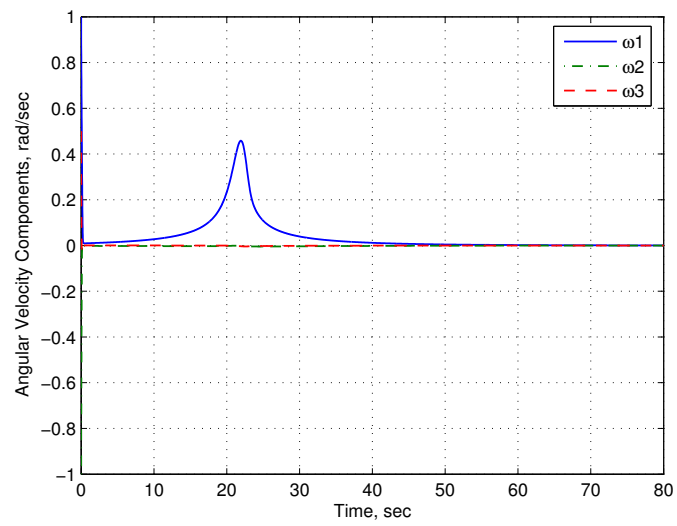

(f) Angular velocity with $\mu=10$

Figure 16: Eigenaxis attitude errors and spacecraft angular-velocity components for gyro scale-factors 0.1 , 1 , and 10 . 


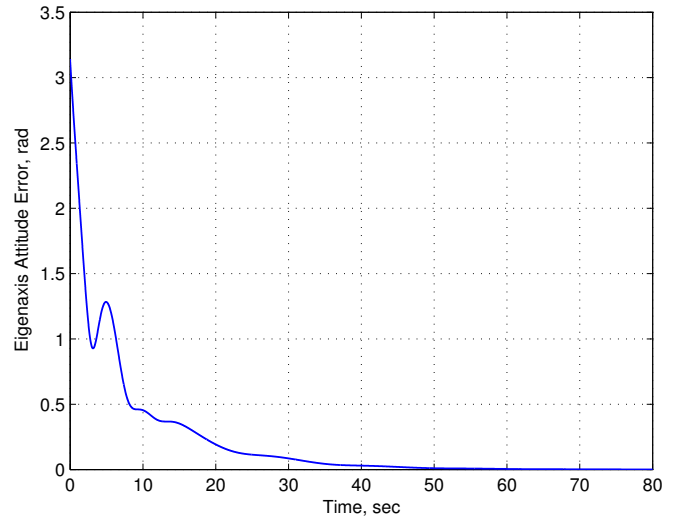

(a) Eigenaxis attitude error with $\mu=0.1$

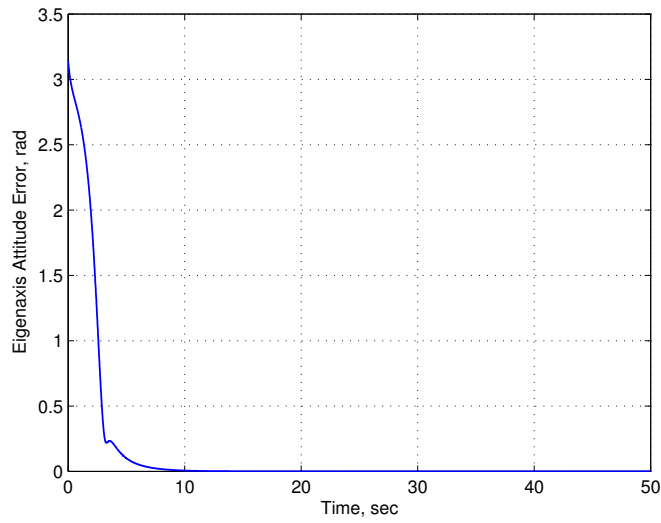

(c) Eigenaxis attitude error with $\mu=1$

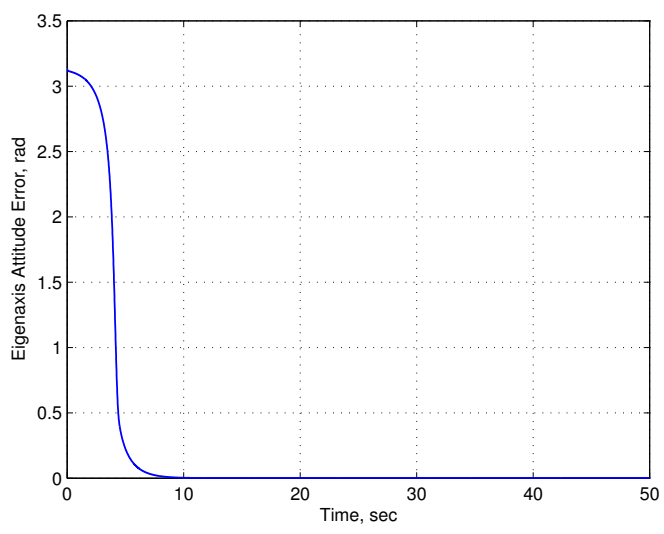

(e) Eigenaxis attitude error with $\mu=10$

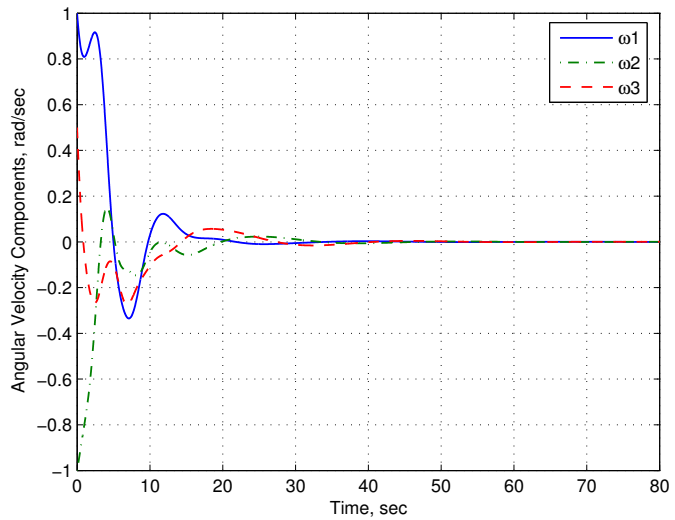

(b) Angular velocity with $\mu=0.1$

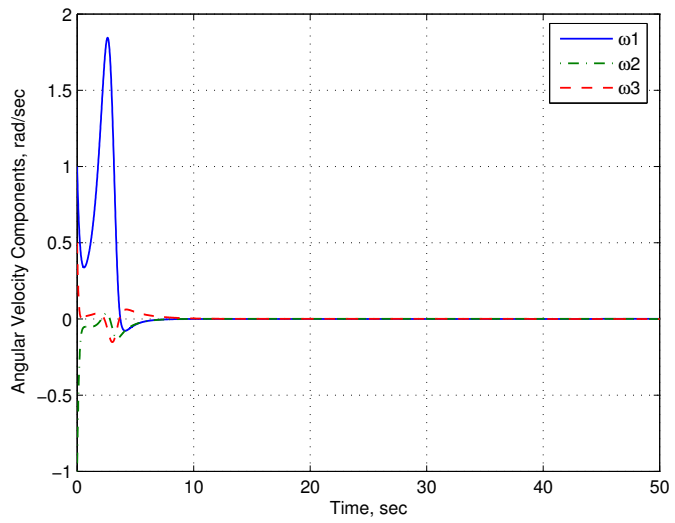

(d) Angular velocity with $\mu=1$

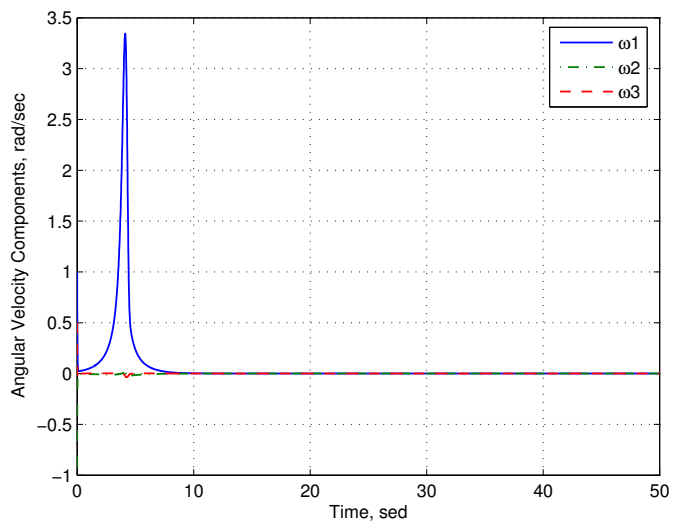

(f) Angular velocity with $\mu=10$

Figure 17: Eigenaxis attitude errors and spacecraft angular-velocity components for actuator scale-factors $0.1,1$, and 10 . 


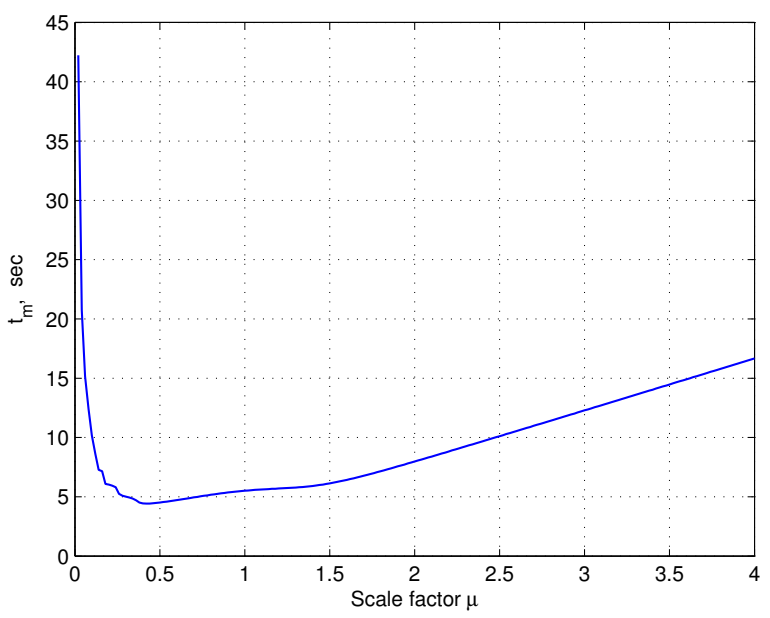

Figure 18: Performance metric versus sensor scale-factor.

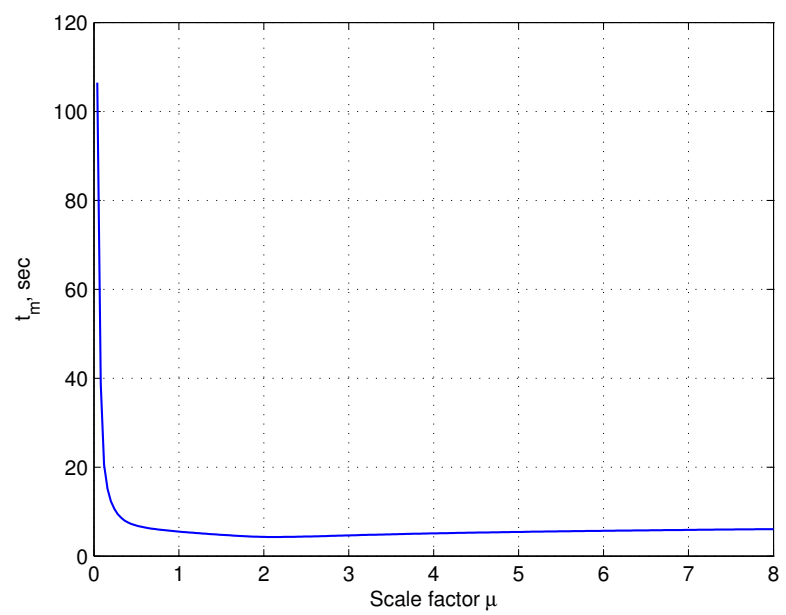

Figure 19: Performance metric versus actuator scale-factor.

\section{Sensor Noise}

We now present a numerical study of the robustness of the control law (38) to gyro noise. We simulate the effects caused by constant, sinusoidal, and white noise added to the gyro measurement as given in (20).

\section{VII.A. Constant Noise}

Assume the gyro noise is given by

$$
v_{\text {rate }}=\alpha[1,1,1]^{\mathrm{T}}
$$

where $\alpha$ is a constant scalar. Figure 20 shows the eigenaxis attitude errors for various values of $\alpha$, and Figure 21 shows angular-velocity components. Tracking performance degrades gracefully as the level of gyro noise increases. 


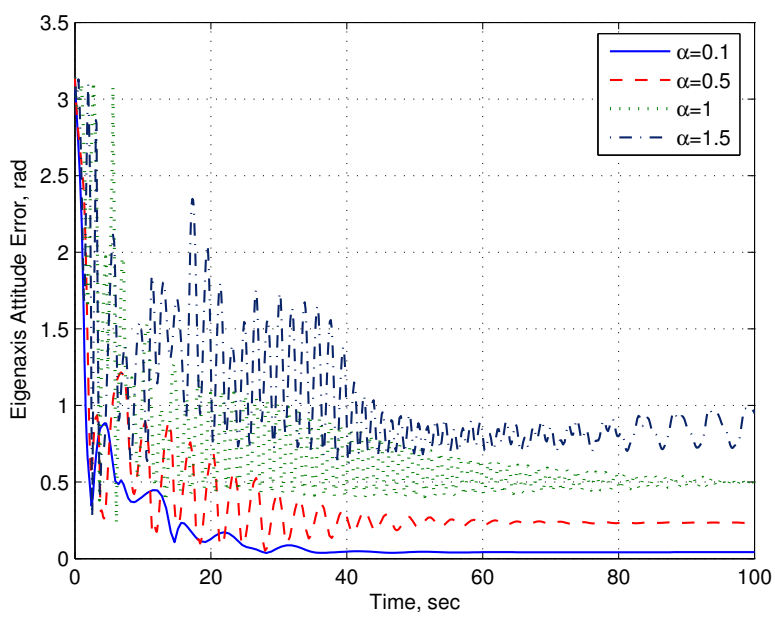

Figure 20: Eigenaxis attitude error for constant noise in the gyro measurement.

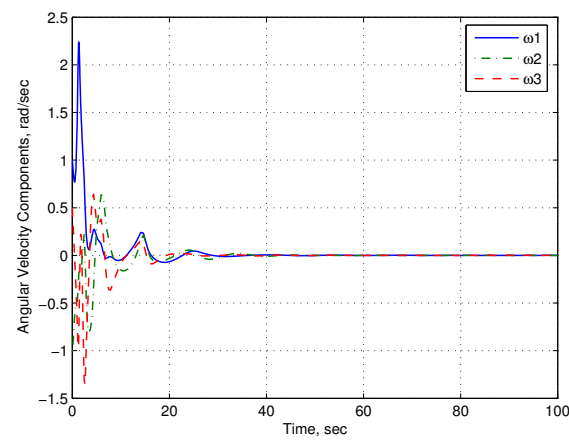

(a) Angular-velocity with $\alpha=0.1$

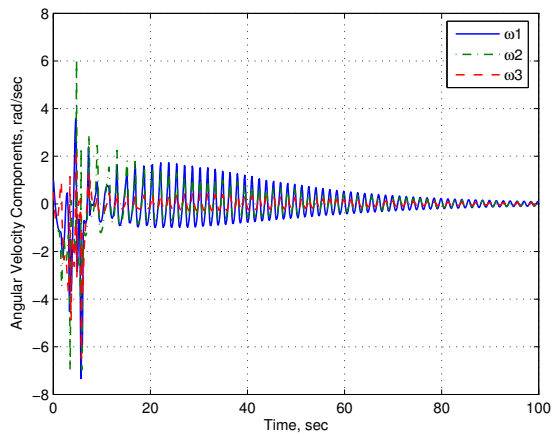

(c) Angular-velocity with $\alpha=1$

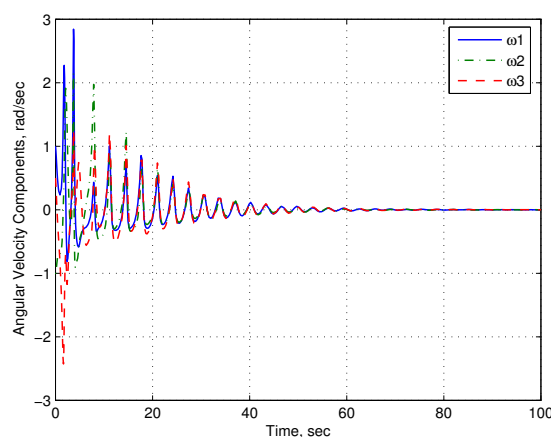

(b) Angular-velocity with $\alpha=0.5$

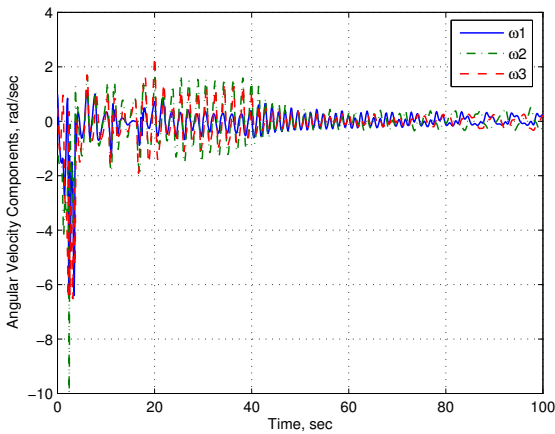

(d) Angular-velocity with $\alpha=1.5$

Figure 21: Angular-velocity components for constant noise in the gyro measurement. 


\section{VII.B. Sinusoidal Noise}

We now assume that the gyro noise is given by

$$
v_{\text {rate }}=(\beta \sin t)[1,1,1]^{\mathrm{T}},
$$

where $\beta$ is a constant scalar. Figures $22-23$ show simulation results for various values of $\beta$. Tracking performance degrades gracefully as the level of gyro noise increases.

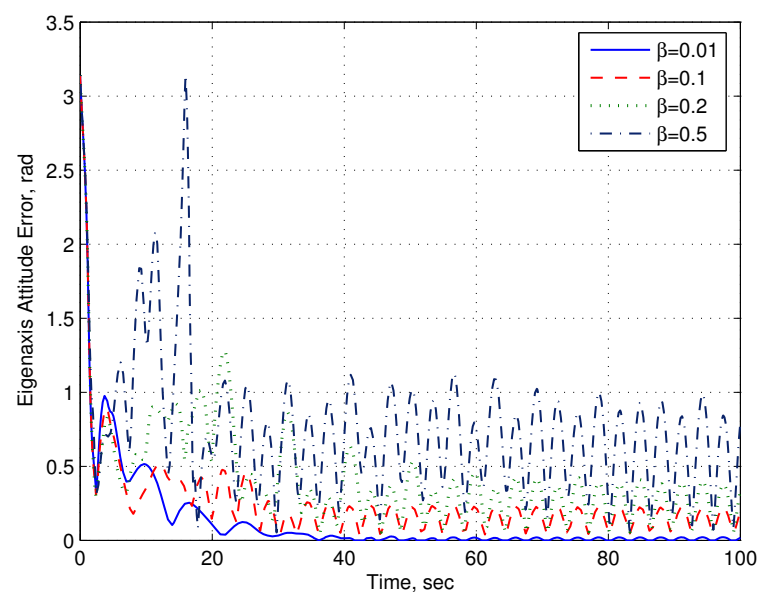

Figure 22: Eigenaxis attitude errors for sinusoidal noise of various amplitude in the gyro measurement.

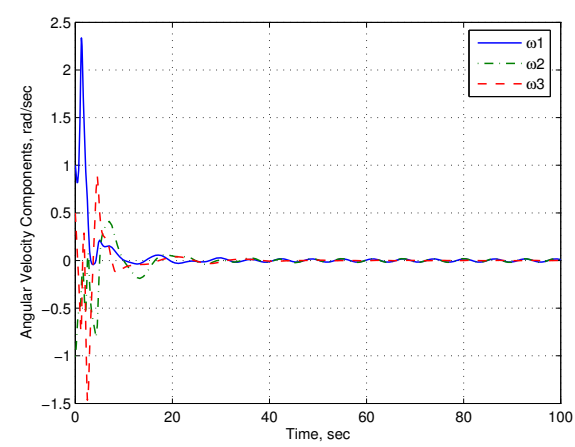

(a) Angular velocity with $\beta=0.01$

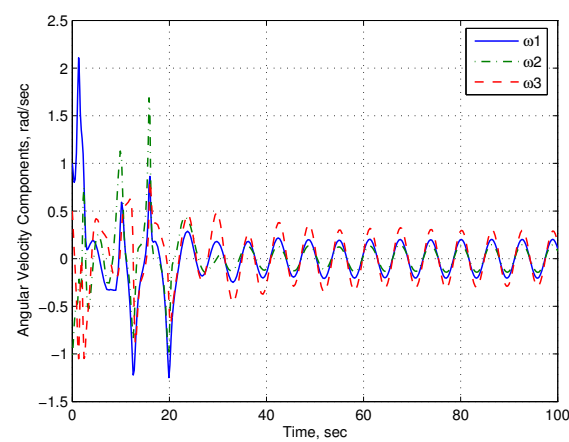

(c) Angular velocity with $\beta=0.2$

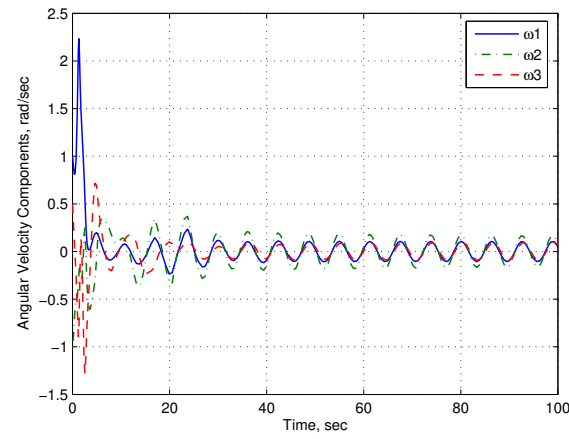

(b) Angular velocity with $\beta=0.1$

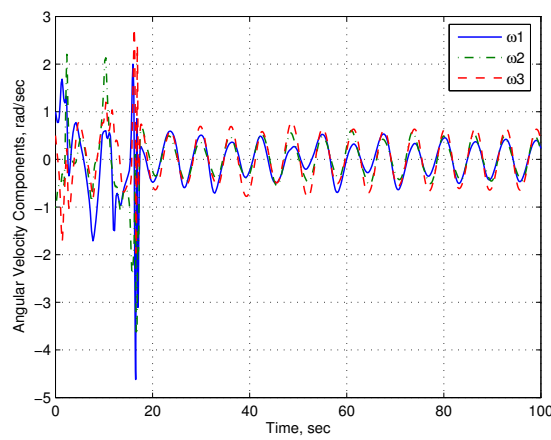

(d) Angular velocity with $\beta=0.5$

Figure 23: Angular-velocity components for sinusoidal noise of various amplitude in the gyro measurement. 
We now assume that the gyro noise is given by

$$
v_{\text {rate }}=\left(0.2 \sin \omega_{\text {noise }} t\right)[1,1,1]^{\mathrm{T}},
$$

where $\omega_{\text {noise }}$ is a constant scalar with units of $[\mathrm{sec}]^{-1}$. Figures $24-25$ show simulation results for various values of $\omega_{\text {noise }}$. Attitude tracking performance improves as the frequency of the gyro noise increases.

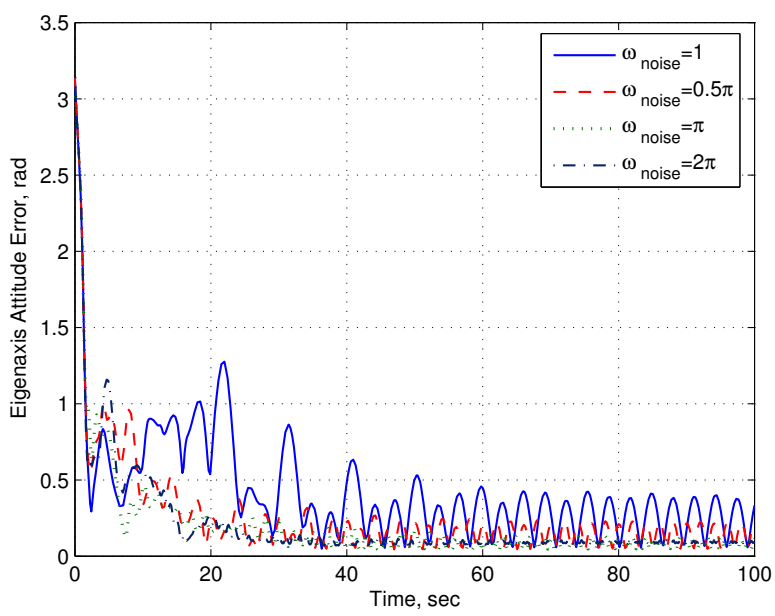

Figure 24: Eigenaxis attitude errors for sinusoidal noise of various frequencies in the gyro measurement.

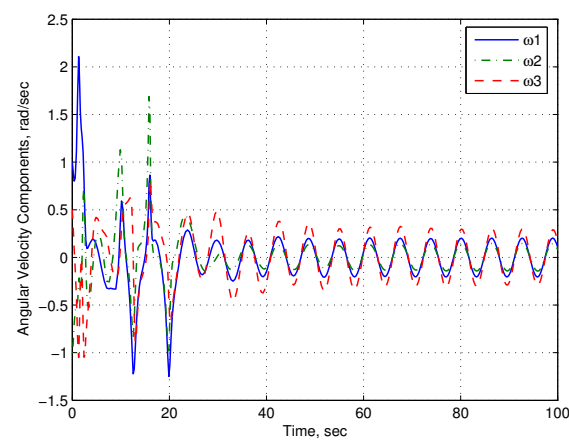

(a) Angular velocity with $\omega_{\text {noise }}=1$

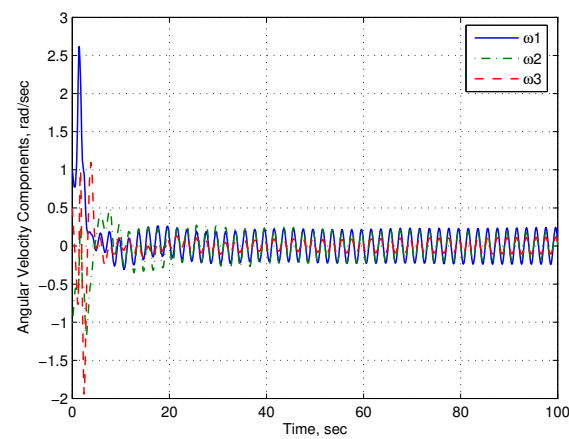

(c) Angular velocity with $\omega_{\text {noise }}=\pi$

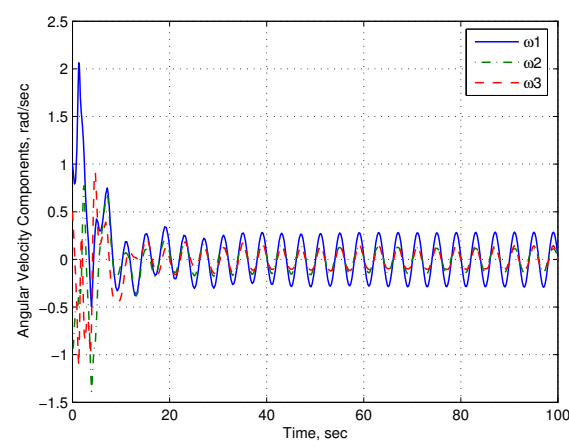

(b) Angular velocity with $\omega_{\text {noise }}=0.5 \pi$

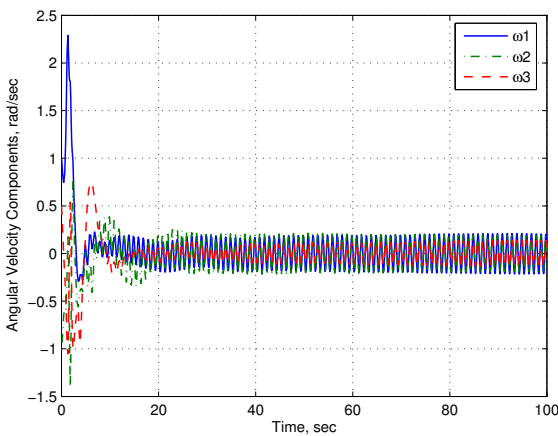

(d) Angular velocity with $\omega_{\text {noise }}=2 \pi$

Figure 25: Angular-velocity components for sinusoidal noise of various frequencies in the gyro measurement. 


\section{VII.C. White Gaussian Noise}

Finally, we create white gaussian noise $W$ with mean 0 and variance 1 , and assume that the gyro noise is given by

$$
v_{\text {rate }}=\gamma W[1,1,1]^{\mathrm{T}},
$$

where $\gamma$ is a constant scalar. Figures 26-27 show simulation results for various values of $\gamma$. Tracking performance degrades significantly in the presence of large amplitude white noise.

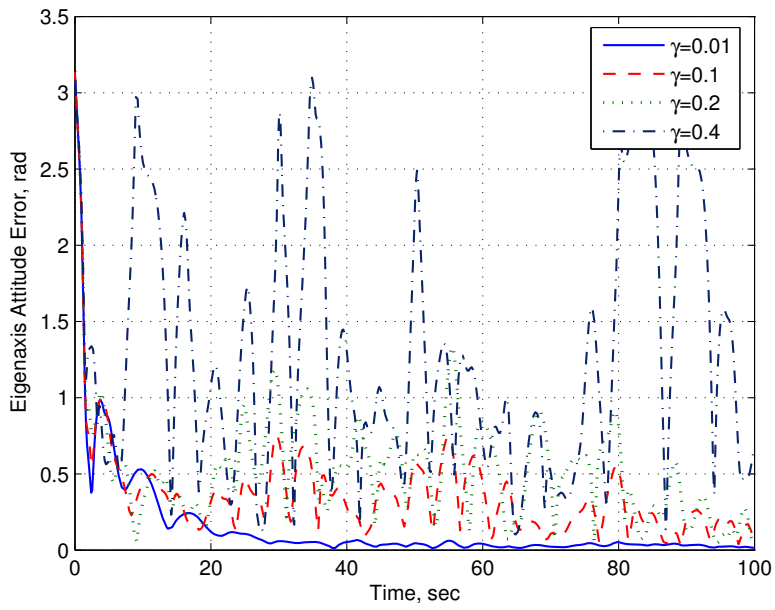

Figure 26: Eigenaxis attitude errors for white noise in the gyro measurement.

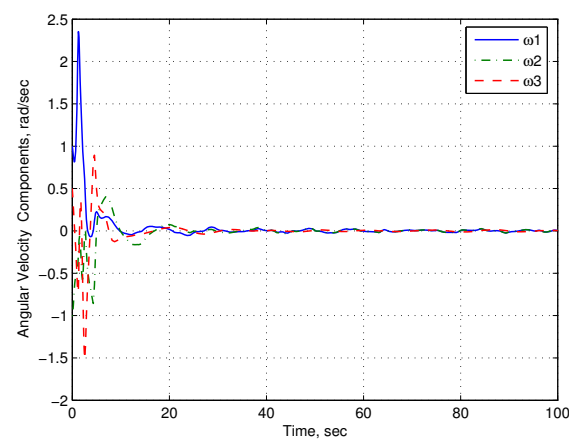

(a) Angular velocity with $\gamma=0.01$

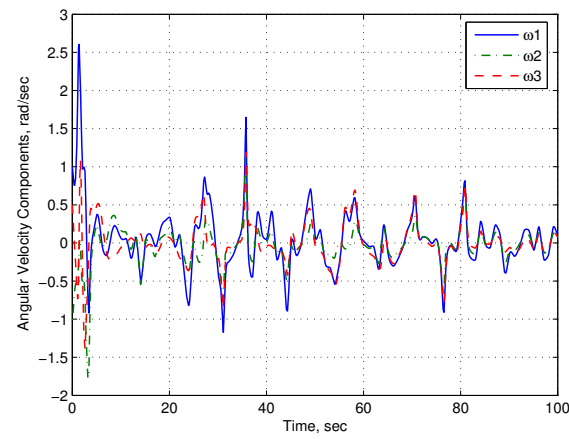

(c) Angular velocity with $\gamma=0.2$

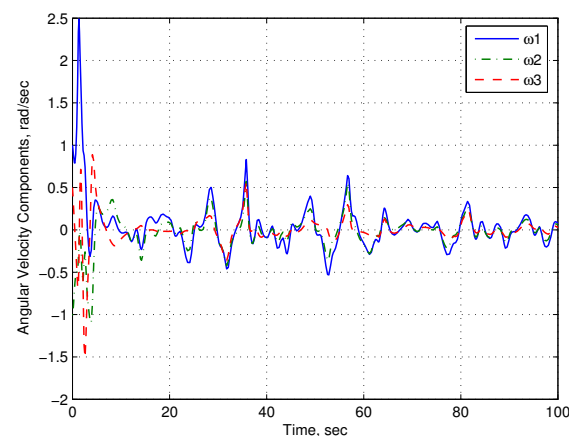

(b) Angular velocity with $\gamma=0.1$

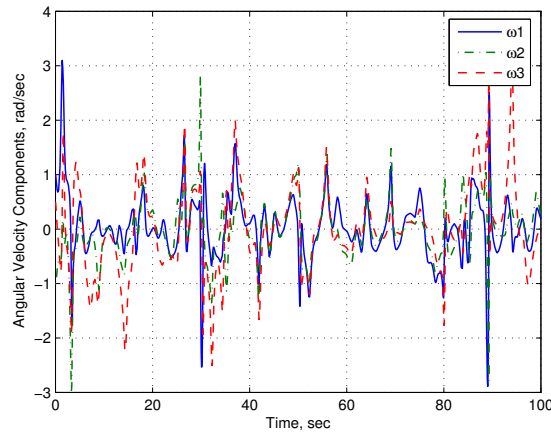

(d) Angular velocity with $\gamma=0.4$

Figure 27: Angular-velocity components for white noise in the gyro measurement. 


\section{Conclusion}

We extended the control laws of ref. ${ }^{1}$ to the case of reaction-wheel actuation. We do not require knowledge of the spacecraft's inertia, only that of the spin axis for each wheel. These results have practical advantages relative to previous controllers that 1) require exact or approximate inertia information or 2) are based on attitude parameterizations such as quaternions that require discontinuous control laws or fail to be physically consistent (that is, specify different control torques for the same physical orientation).

We simulated these controllers for various slew and spin maneuvers. Additionally, we explicitly considered sensor and actuator scale-factor uncertainty and found that for positive scale-factors, controller (30) remains asymptotically stable. Finally, we presented a numerical study on the effect of gyro measurement noise and found that controller (38) is fairly robust to various sources of noise.

\section{References}

${ }^{1}$ A. Sanyal, A. Fosbury, N. Chaturvedi, and D. S. Bernstein, "Inertia-Free Spacecraft Attitude Tracking with Disturbance Rejection and Almost Global Stabilization," AIAA J. Guid. Contr. Dyn., Vol. 32, pp. 1167-1178, 2009.

${ }^{2}$ Ahmed, J., Coppola, V. T., and Bernstein, D. S., "Asymptotic Tracking of Spacecraft Attitude Motion with Inertia Matrix Identification," AIAA Journal of Guidance, Control, and Dynamics, Vol. 21, pp. 684-691, 1998.

${ }^{3}$ Wie, B., and Barba, P. M., "Quaternion Feedback for Spacecraft Large Angle Maneuvers," AIAA Journal of Guidance, Control, and Dynamics, Vol. 8, pp. 360-365, 1985.

${ }^{4}$ Joshi, S. M., Kelkar, A. G., and Wen, J. T., "Robust attitude stabilization using nonlinear quaternion feedback," IEEE Transactions on Automatic Control, Vol. 40, pp. 1148-1161, 1995.

${ }^{5}$ Bhat, S. P., and Bernstein, D. S., "A Topological Obstruction to Continuous Global Stabilization of Rotational Motion and the Unwinding Phenomenon," Systems and Control Letters, Vol. 39, pp. 63-70, 2000.

${ }^{6}$ Cortes, J., "Discontinuous Dynamic Systems," IEEE Control Systems Magazine, Vol. 28, pp. 36-71, June 2008.

${ }^{7}$ Crassidis, J. L., Vadali, S. R., and Markley, F. L., "Optimal Variable-Structure Control Tracking of Spacecraft Maneuvers," AIAA Journal of Guidance, Control, and Dynamics, Vol. 23, pp. 564-566, 2000.

${ }^{8}$ Mayhew, C. G., Sanfelice, R. G., and Teel, A. R., "Robust Global Asymptotic Stabilization of a 6-DOF Rigid Body by Quaternion-based Hybrid Feedback," Proc. IEEE CDC, pp. 1094-1099, 2009.

${ }^{9}$ Arambel, P. O., Manikonda, V., and Mehra, R. K., "Spacecraft Attitude Tracking in the Presence of Input Magnitude Constraints," Proceedings of the American Control Conference, pp. 4082-4086, June 2000.

${ }^{10}$ Boskovic, J. D., Li, S.-M., and Mehra, R. K., "Robust Adaptive Variable Structure Control of Spacecraft Under Control Input Saturation," AIAA Journal of Guidance, Control, and Dynamics, Vol. 24, pp. 14-22, 2001.

${ }^{11}$ Banga, H., Tahka, M.-J., and Choib, H.-D., "Large Angle Attitude Control of Spacecraft with Actuator Saturation," Control Engineering Practice, Vol. 11, pp. 989-997, 2003.

${ }^{12}$ Hughes, P. C., Spacecraft Attitude Dynamics, Wiley, 1986; reprinted by Dover, 2008, page 17.

${ }^{13}$ Sanyal, A. K., Lee, T., Leok, M., and McClamroch, N. H., "Global Optimal Attitude Estimation Using Uncertainty Ellipsoids," Systems and Control Letters, Vol. 57, pp. 236-245, 2008.

${ }^{14}$ Levine, W. S., Control Systems Applications, CRC Press, Inc., Boca Raton, FL, USA, 1999. 